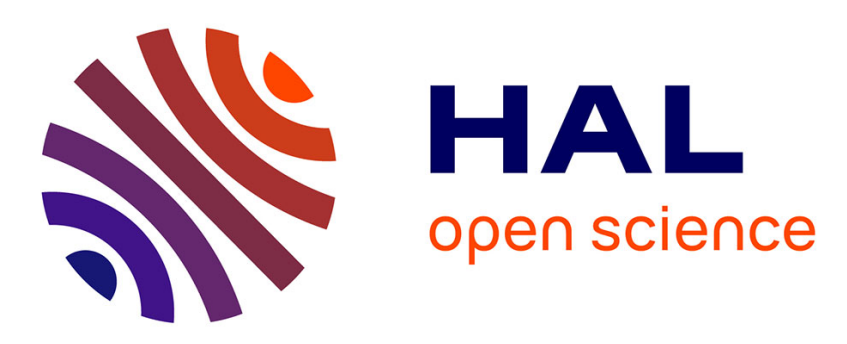

\title{
Hindbrain Interneurons and Axon Guidance Signalling Critical for Breathing
}

Julien Bouvier, Muriel Thoby-Brisson, Nicolas Renier, Véronique Dubreuil, John Ericson, Jean Champagnat, Alessandra Pierani, Alain Chedotal, Gilles Fortin

\section{To cite this version:}

Julien Bouvier, Muriel Thoby-Brisson, Nicolas Renier, Véronique Dubreuil, John Ericson, et al.. Hindbrain Interneurons and Axon Guidance Signalling Critical for Breathing. Nature Neuroscience, 2010, 13 (9), pp.1066-74. 10.1038/nn.2622 . hal-00561814

\section{HAL Id: hal-00561814 https://hal.science/hal-00561814}

Submitted on 2 Feb 2011

HAL is a multi-disciplinary open access archive for the deposit and dissemination of scientific research documents, whether they are published or not. The documents may come from teaching and research institutions in France or abroad, or from public or private research centers.
L'archive ouverte pluridisciplinaire HAL, est destinée au dépôt et à la diffusion de documents scientifiques de niveau recherche, publiés ou non, émanant des établissements d'enseignement et de recherche français ou étrangers, des laboratoires publics ou privés. 


\section{NN-A32651A}

\section{Hindbrain Interneurons and Axon Guidance Signalling Critical for}

Breathing

Bouvier $\mathrm{J}^{1}$, Thoby-Brisson $\mathrm{M}^{{ }^{*}}$, Renier $\mathrm{N}^{2,}{ }^{3,} 4^{*}$, Dubreuil $\mathrm{V}^{5}$, Ericson $\mathrm{J}^{6}$, Champagnat $\mathrm{J}^{1}$, Pierani $A^{7 \#}$, Chédotal $A^{2,3,4 \#}$ and Fortin $G^{1 \#}$

1 Institut de Neurobiologie Alfred Fessard, CNRS UPR 3294, Gif-sur-Yvette, France

${ }^{2}$ INSERM, U968, Paris F-75012, France

${ }^{3}$ UPMC Univ Paris 06, UMR_S968, Institut de la Vision, Paris F-75012, France

${ }^{4}$ CNRS, UMR_7210, Paris F-75012, France

${ }^{5}$ CNRS, UMR 7216, Université Paris 7, France

${ }^{6}$ Cell and Molecular Biology, Karolinska Institute, Stockholm, Sweden

${ }^{7}$ Institut Jacques Monod, CNRS UMR 7592, Université Paris 7, France

\#co-corresponding authors

*equal contributions

\section{Corresponding author:}

pierani.alessandra@ijm.univ-paris-diderot.fr

alain.chedotal@inserm.fr

gilles.fortin@inaf.cnrs-gif.fr

Summary: 127 words

Figures: 7

Supplementary Figures: 3

Supplementary Video: 1 


\section{SUMMARY}

Breathing is a bilaterally synchronous behavior that relies on a respiratory rhythm generator located in the brainstem. An essential component of this generator is the preBötzinger complex (preBötC) that paces inspirations. Little is known about the developmental origin of interneuronal populations forming the preBötC oscillator network. We show that the homeobox gene Dbx1 controls the fate of glutamatergic interneurons required for preBötC rhythm generation in the mouse embryo. We also show that a conditional inactivation in Dbx1-derived cells of the Roundabout receptor 3 (Robo3) gene, necessary for axonal midline crossing, results in left-right de-synchronization of the preBötC oscillator. Together, these findings identify Dbx1-derived interneurons as the core rhythmogenic elements of the preBötC oscillator, and Robo3-dependent guidance signaling in these cells as a requirement for bilaterally synchronous activity.

\section{INTRODUCTION}

Breathing is a motor behavior generated by a respiratory rhythm generating network located in the brainstem that produces a synchronous bilateral drive onto cranial and spinal populations of motor neurons that innervate cranial, thoracic and abdominal respiratory muscles ${ }^{1}$.

A functional respiratory network is necessary for survival at birth, and respiratory-like movements and respiratory-like network activity in the brainstem are detected before birth. In fetal and newborn rodents, the respiratory rhythm generating network consists of two distinct but functionally coupled oscillators: the retrotrapezoid nucleus/parafacial respiratory group (RTN/pFRG), and the preBötzinger Complex (PreBötC). Previous work in the mouse embryo revealed that the embryonic parafacial (e-pF) oscillator (the forerunner of the RTN/pFRG) and the preBötC emerge independently at distinct anterior-posterior positions close to the facial branchiomotor nucleus and to the nucleus ambiguus (n.a.), respectively ${ }^{2}$. The e-pF and preBötC become active sequentially, first the e-pF at E14.5 and then, at E15.5, the preBötC oscillator with which the e-pF couples ${ }^{2}$. As recently shown, in multiple mutant mice in which the RTN/pFRG does not form ${ }^{2-4}$ one of its roles is to increase the frequency of the respiratory rhythm generating network through entrainment of the preBötC that in turn controls the motor output.

Little is known about the developmental origin and molecular determinants of preBötC neurons. The preBötC identified first functionally ${ }^{5}$ is anatomically characterized as a collection of glutamatergic neurons in the ventral respiratory column, located beneath the n.a., that express the neurokinin type1 receptor $(\mathrm{NK} 1 \mathrm{R})^{6}$ and somatostatin (Sst) ${ }^{7}, 8$. Accordingly, chemical ablation of NK1 ${ }^{9}$ preBötC neurons or genetic/viral silencing of Sst-positive preBötC neurons ${ }^{10}$ stop breathing in rats.

Mafb mutants are the only mouse mutants in which the preBötC is disrupted ${ }^{11}$, but the early widespread expression of Mafb in the caudal hindbrain leaves open the possibility that impaired development of other structures contributes to the 
lethal respiratory phenotype of these mutants. We have previously reported an "absent breathing" phenotype associated to a defective preBötC oscillator after disruption of the type 2 vesicular glutamate transporter in the conditional Nestin::Cre; Vglut2 ${ }^{\text {lox/lox }}$ mutant $^{12}$. However, the global and non selective disruption of glutamatergic transmission in these mice precluded evaluation of the specific contribution of preBötC neurons to the breathing deficit.

The bilateral synchronization of the preBötC is likely to rely on commissural projections ${ }^{7}, 8,13,14$ including those arising from glutamatergic $\mathrm{NK}^{7} \mathrm{R}^{7}$ and $\mathrm{Sst}^{7,8}$ neurons. However, the molecular mechanisms underlying commissural connectivity of the preBötC have not been addressed. Several recent studies have shown that in the mammalian hindbrain and spinal cord, the development of many commissural projections is controlled by the Roundabout 3 receptor (Robo3; also known as Rig1) ${ }^{15-19}$.

In vertebrates, the intricate spatial order of diverse neuronal types, essential to the subsequent formation of functional circuits arises from transcriptional codes coordinating anterior-posterior and dorsal-ventral patterning of the neural tube $e^{20,}$ 21. Distinct classes of interneurons and motor neurons differentiate in relation to the expression of homeodomain proteins within progenitor domains at defined locations in the neural tube ${ }^{22}$. In mammals, the role of transcriptionally identified commissural interneurons in regulating the function of neural networks has been best characterized at the level of the central pattern generator that controls locomotion in the spinal cord ${ }^{23}$. There, the absence of so-called ventral ( $\mathrm{V}$ ) V0 or V3 classes of interneurons that originate respectively from progenitor cells (P) P0 and $\mathrm{P} 3$ that express the $\mathrm{Dbx} 1$ and $\mathrm{Nkx} 2.2 / \mathrm{Sim} 1$ genes, correlates with impaired left/right alternation ${ }^{24}$ and impaired robustness of the symmetrical left/right patterning of locomotor activity ${ }^{19}$. By analogy with the spinal cord, and considering that V3 interneurons are absent at the level of the hindbrain, where the P3 progenitor domain is replaced by a PVMN domain that gives rise to visceral motor neurons ${ }^{25-28}$, we considered the progeny of Dbx1-expressing $\left(D b \times 1^{+}\right)$P0 progenitors to be candidate preBötC neurons and investigated the respiratory outcome of a null mutation of the Dbxl gene ${ }^{29}$.

In this study, we have used several transgenic mouse lines to identify the origin of preBötC neurons and the molecular identity of their embryonic progenitors. We show that rhythm generation by the preBötC depends on the homeobox gene Dbx1, expressed in the PO neural progenitor domain of the hindbrain ${ }^{29,30}$. Dbx1 ablation completely silences the preBötC and causes a massive loss of preBötC glutamatergic neurons, including all $\mathrm{NK}_{1} \mathrm{R}^{+}$and $\mathrm{Sst}^{+}$interneurons. Dbx1dependent neurons ensure not only rhythmicity but also bilateral synchrony of the preBötC, where Robo3 signaling is required for these cells to project to the contralateral preBötC. The selective disruption of Robo3 in the descendants of $\mathrm{Dbx}^{+}$progenitors results in a preBötC featuring asynchronous left/right rhythmicity. This suggests that the "core preBötC", i.e. the collective of cells insuring its two essential properties (rhythm generation and bilateral synchrony), arises from the $\mathrm{Dbx} 1^{+}$progenitor domain. Thus, $\mathrm{Dbx} 1$, beyond its classical role in 
the regionalization of the neuroepithelium and specification of neuronal subtypes, emerges as a key regulator for the assembly of a vital oscillator network.

\section{RESULTS}

\section{Dbx1 null mutants do not breathe.}

At birth, Dbx1 null (Dbx1 $1^{\text {LacZ/LacZ }) ~ m u t a n t s ~ h a v e ~ a ~ b e a t i n g ~ h e a r t ~ b u t ~ s h o w ~ n o ~}$ breathing movements, turn cyanotic and die within minutes of delivery (Fig. 1). Unlike Dbx1 heterozygous ( $\mathrm{Dbx} 1^{\mathrm{LacZ} /+}$ ) and wildtype littermates who initiate breathing ( $f=80.2 \pm 7.7$ breath/min, $n=10$ from 3 litters) immediately after birth or upon delivery from uterine horns on embryonic (E) day E18.5 (Fig. 1a), all Dbx $1^{\text {LacZ/LacZ }}$ pups (E18.5, $\left.n=7 ; P 0, n=3\right)$ failed to do so (Fig. 1b). At E15.5, the onset of so-called "fetal breathing"2, electrophysiological recordings and calcium imaging on $\mathrm{Dbx} 1^{\text {Lacz/LacZ }}$ transverse preBötC slices $(n=7$, Fig. 1c-f) and whole hindbrain preparations $(n=3$, Fig. 19-j), revealed the absence of respiratory rhythm-like fluorescence changes in the preBötC area (Fig. 1c,d) and activity in the $4^{\text {th }}$ cervical root (C4) where the phrenic nerve exits to innervate the diaphragm (Fig. 1i $\mathbf{j} \mathbf{j}$ ). Furthermore, bath applications of glutamate agonists AMPA ( $1 \mu \mathrm{M}, \mathrm{n}=2)$ and NMDA ( $1 \mu \mathrm{M}, \mathrm{n}=3$, data not shown), or the neuropeptide Substance $P(1 \mu M, n=4)$, failed to initiate preBötC and $C 4$ rhythmic bursting activity (Fig. 1e,f). In contrast, spontaneous rhythmic fluorescence changes in the e-pF oscillator area ( $f=12.9 \pm 0.6$ burst/min, $n=5$ ) were spared in Dbxi ${ }^{\text {Lacz/Lacz }}$ embryos ( $f=15.1 \pm 1.9$ bursts/min, $n=3$, Fig. 1g-j). Hence, although active, the e-pF oscillator was apparently unable to rhythmically drive the respiratory motor outputs in the absence of a functional preBötC oscillator. These observations suggest that the respiratory deficit of $\mathrm{Dbx} 1^{\mathrm{LacZ} / \mathrm{LacZ}}$ mice is central and results from the catastrophic impairment of the preBötC oscillator.

\section{Dbx1-derived interneurons in the preBötc.}

Next we examined the contribution of $\mathrm{Dbx} 1^{+}$progenitors to the preBötC by genetic fate-mapping, using the previously characterized $\mathrm{Dbx} 1^{\mathrm{Lacz} /+} \operatorname{line}^{29}$ in which beta-Galactosidase ( $B-G a l$ ) expression outlasts that of $D b \times 1$, which is turned-off as progenitors exit the cell cycle ${ }^{24}$ (Fig. 2a-d), and a novel Dbx $1^{\text {IRES- }}$ GFP line (see methods) allowing visualization of Dbx1-derived cells in physiological preparations (Fig. 2e-g). In Dbxi $1^{\text {Lacz/t }}$ E15.5 embryos $(n=9)$, double immunostaining for B-Gal and Islet1,2 (Isl1,2) a motor neuronal marker ${ }^{31}$, indicated that $B-\mathrm{Gal}$ positive $\left(B-\mathrm{Gal}^{+}\right)$cells had migrated medially from ventricular positions near the sulcus limitans to populate territories bordering the Isl $1,2^{+}$hypoglossal and dorsal motor vagal nuclei, and radially to a occupy a ventro-lateral area in the vicinity of the Isl $1,2^{+}$n.a. ${ }^{32}$ (Fig. $\mathbf{2 c}$ ). The position of

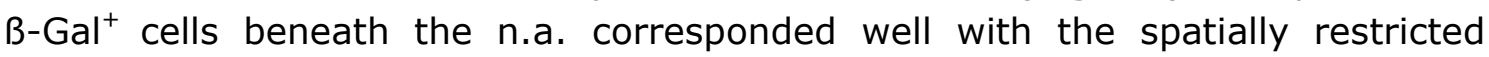
domain where, in transverse medullary slice preparations loaded with Calcium green-1 AM, spontaneous rhythmic fluorescence changes characterize the activity of the preBötC oscillator (Fig. 2d) ${ }^{32}$. Imaging in this region at cellular resolution in E15.5 Dbx $1^{\text {IRES-GFP }}$ preBötC slices, and selecting $\mathrm{GFP}^{+}$cells for which 
registration with the optical signal was obvious, showed synchronous rhythmic fluorescent changes for 54/64 GFP ${ }^{+}$cells from 5 slices (Fig. 2h,i). Furthermore, the rhythmic status of Dbx1-derived cells in the vicinity of the n.a. was confirmed by whole cell recordings (Fig. $\mathbf{2} \mathbf{j}, \mathbf{I}$ ). Seven of ten recorded $\mathrm{GFP}^{+}$neurons (5 slices) spontaneously discharged rhythmic bursts of action potentials (Fig. 2k) and, in voltage clamp mode, showed transient volleys of inward synaptic currents in phase with population bursting activity recorded from the contralateral preBötC (Fig. 2l). Thus Dbx1-derived cells feature functional signatures of preBötC neurons.

To estimate the birthdate of Dbx1-derived cells of the preBötC area, we performed single-pulse BrdU injections on pregnant dams from E9.5 to E14.5 (Supplementary Fig. 1d-h). Results show that the majority of $B-\mathrm{Gal}^{+}$cells in the preBötC area are born between E10.5 (37.8 \%) and E11.5 (41.9\%). Virtually no $\mathrm{B}_{-\mathrm{Gal}^{+}}{ }^{\mathrm{BrdU}}{ }^{+}$cells were found following injections at E9.5 $(1.6 \%)$, E12.5 (2.8\%) or E13.5 (0.9\%) (Supplementary Fig. 1i). The number and

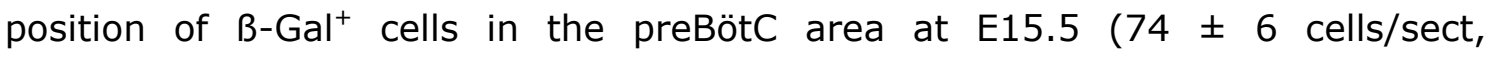
$\mathrm{n}=9$ embryos) and E18.5 (79 \pm 3 cells/sect, $\mathrm{n}=5$ embryos, Supplementary. Fig. 1a-c) were similar, confirming their final settling in the preBötC at $E 15.5^{32}$ and the perdurance of $B-G a l$ protein expression ${ }^{29}$ at least up to E18.5.

We used triple immunostaining for $B-G a l, ~ I s l 1,2$ (Fig. 3a) and either Sst or NK1R, two established markers for subsets of preBötC neurons (Fig. $\mathbf{3 b}, \mathbf{c}$ ), to further define the molecular phenotype of $\beta-\mathrm{Gal}^{+}$cells (Fig. 3d-i). We found that $91 \%$ of $\mathrm{Sst}^{+}$neurons co-expressed $\mathrm{B}-\mathrm{Gal}$ and were thus derived from $\mathrm{Dbx} 1^{+}$ progenitors (Fig. 3d,e,t). Conversely, $\mathrm{Sst}^{+}$neurons accounted for about $13 \%$ of B-Gal ${ }^{+}$cells of the preBötC area (Table 1). Because of the predominant staining of cellular processes by anti-NK1R antibodies (Fig. 3f,g), B-Gal expression in $\mathrm{NK}_{1} \mathrm{R}^{+}$somas, although verified in all cases, depended on few observations and should be considered as a qualitative indication that the majority of $\mathrm{NK} \mathrm{R}^{+}$cells also derived from $\mathrm{Dbx} 1^{+}$progenitors.

Glutamatergic neurotransmission is required for rhythm generation in the preBötC $^{5,33,34}$ and the type 2 vesicular glutamate transporter (Vglut2) therein for the loading of synaptic vesicles ${ }^{12}$. Therefore, we next investigated whether Dbx1derived PreBötC neurons were glutamatergic. Combining B-Gal immunostaining and in situ hybridization for $\mathrm{Vglut}^{3}$ revealed that $82 \%$ of $\mathrm{B}-\mathrm{Gal}^{+}$cells in the preBötC area were Vglut2 $^{+}$(Fig. 3h,i and Table 1), including most (96\%) Sst ${ }^{+}$ neurons (Fig. $\mathbf{3 j}$ ). These data suggest that a subset of $\mathrm{Dbx} 1^{+}$progenitors gives rise between E10.5-11.5 to glutamatergic interneurons displaying, at later embryonic stages, the appropriate locations, neurochemical identities and functional properties of constitutive neurons of the preBötC.

\section{Disruption of preBötC molecular identity.}

The absence of respiratory-like rhythmic activity in $\mathrm{Dbx} 1^{\mathrm{LacZ} / \mathrm{LacZ}}$ mutant could be caused by an absence or a substantial depletion of preBötC neurons. To test this hypothesis we counted the number of $\mathrm{B}-\mathrm{Gal}^{+}, \mathrm{NK} \mathrm{R}^{+}$and $\mathrm{Sst}^{+}$neurons in control and Dbx $1^{\text {LacZ/LacZ }}$ mutants. 
The spatial distribution and total number of $B-\mathrm{Gal}^{+}$cells in the preBötC area were similar in $\mathrm{Dbx} 1^{\mathrm{Lacz} /+}\left(74.6 \pm 6.0\right.$ cells/section; 9 embryos) and in Dbx $1^{\text {Lacz/Lacz }}$ mice (71.5 \pm 6.5 cells/section; 5 embryos), suggesting that the absence of the Dbx1 protein in progenitors affected neither the survival nor the migration of Dbx1-derived neuronal progenies in the preBötC (Fig. 3a,k).

However, in $\mathrm{Dbx} 1^{\text {Lacz/LacZ }}$ mutants, there was an abnormal differentiation of the preBötC shown most conspicuously by the complete absence of Sst and NK1R immunoreactivity (Fig. 3I, $\mathbf{m}$ ). This resulted from loss of expression of the markers in $\mathrm{B}-\mathrm{Gal}^{+}$cells (Fig. 3n-q,u).

Most importantly, the impairment of the differentiation of Dbx1-derived cells in the mutants extended to the loss of Vglut2 expression in $90 \%$ of $\mathrm{B}_{-\mathrm{Gal}^{+}}$cells (Fig. 3r,s,u, Table1). The latter change is probably the cause of the preBötC functional deficit.

\section{The PreBötC originates in ventral $\mathrm{Dbx1^{+ }}$ domain.}

In the developing spinal cord, the $\mathrm{Dbx} 1^{+}$progenitor domain is subdivided along the dorsoventral axis into dorsal and ventral subdomains by their respective expression and lack of $P a x 7^{24,29}$. To establish whether such a partitioning of Dbx1-expressing progenitors exists in the hindbrain and accordingly allocate the preBötC progenitors, we performed Pax3/7 immunostaining in $\mathrm{Dbx} 1^{\text {Lacz/+ }}$ mice and analyzed the conditional Pax7::Cre; Dbx $1^{\text {DTA }}$ mutant where only Pax $7^{+}, \mathrm{Db} \times 1^{+}$ progenitors express the diphtheria toxin and consequently die (Fig. 4). In E10.5 Dbx1 $1^{\text {LacZ/+ }}$ embryos, dorsal but not ventral Dbx $1^{+}$progenitors co-expressed Pax 7 as in the spinal cord (Fig. 4a). In Pax7::Cre; Dbx1 ${ }^{\text {DTA }}$ mice, although the dorsal $\mathrm{Db} \times 1^{+}$domain of progenitors was effectively missing (Fig. 4a-c), neither the functional alterations nor the molecular changes identified in Dbx $1^{\text {LacZ/LacZ }}$ mutants were observed. In transverse slices, rhythmicity of the preBötC was maintained (Fig. 4d) and was generated at a frequency ( $f=4,7 \pm 0,9$ burst/min, $n=7$ ) similar to control preparations (wild-type : $f=4,5 \pm 0,4$ burst/min, $\mathrm{n}=26)$. In addition, $\mathrm{Sst}^{+}$and Vglut2 $^{+}$cells were found in numbers (Sst $^{+}: 7.7 \pm 1.0$ cells/sect; Vglut2 ${ }^{+}: 87.4 \pm 4.2$ cells/sect, 3 embryos) similar to those found in $\mathrm{Dbx} 1^{\text {LacZl+ }}$ embryos (Sst ${ }^{+}: 9.4 \pm 1.2$ cells/section; Vglut2 ${ }^{+}: 80.0 \pm$ 3.2 cells/section, 4 embryos, Fig. 4e,f). Furthermore, qualitatively, NK1R expression was also spared in the Pax7::Cre; Dbx $1^{\text {DTA }}$ mutants (Fig. 4g). To further support the idea that $\mathrm{Dbx} 1$-derived $\mathrm{Sst}^{+}, \mathrm{NK}_{1} \mathrm{R}^{+}$and $\mathrm{Vglut2}^{+}$cells of the preBötC area originate from the ventral subdomain of $\mathrm{Dbx1^{+ }}$ progenitors we investigated comparatively the outcome of the Dbx1 mutations on $\mathrm{Evx} 1^{+}$cells thought to arise from the $\mathrm{Dbx} 1^{+}$ventral progenitor subdomain at the spinal level $^{24,29}$. As shown in Figure 4a-c, at E10.5 in the hindbrain, Evx1 ${ }^{+}$cells were confined to lateral positions of the ventral $\mathrm{Dbx} 1^{+} / \mathrm{Pax} 7^{-}$subdomain (Fig. 4a) and were preserved in Pax7::Cre; Dbx1 ${ }^{\text {DTA }}$ embryos (Fig. 4c). Unexpectedly, later in development, at $\mathrm{E} 15.5$, almost half ( $46 \%$ ) of Evx1 cells present in the preBötC area were found not to derive from $\mathrm{Dbx} 1^{+}$progenitors as they neither coexpressed $B-G a l$ nor were found affected in $D b x 1^{\text {LacZ/+ }}$ and $D b x 1^{\text {LacZ/LacZ embryos }}$ (Fig. 4h,i). The complementing half of Evx1 ${ }^{+}$cells however, contributed to about 
a third (31 \%) of the $B-\mathrm{Gal}^{+}$cell population of the preBötC area (Fig. $4 \mathbf{h}$ and Table 1) and almost all (98\%) expressed Vglut2 (Fig. 4l-o). Since the full complement of Evx1 $1^{+}$cells was maintained in Pax7::Cre; Dbx $1^{\text {DTA }}$ embryos (Fig. $\mathbf{4 j , k}$ ) while Evx1-expression was lost in $B-\mathrm{Gal}^{+}$cells of the $\mathrm{Dbx} 1^{\text {LacZ/LacZ }}$ embryos (Fig. 4i,k), we conclude that $\mathrm{Sst}^{+}$and $\mathrm{NK} \mathrm{R}^{+}$cells, like $\mathrm{Evx} 1^{+} / \beta-\mathrm{Gal}^{+}$cells, are glutamatergic preBötC interneurons arising from ventral $\mathrm{Dbx} 1^{+}$progenitors.

As expected from the functional conservation of the e-pF oscillator, lineage tracing experiments in Dbxi $1^{\text {Lacz/+ }}$ embryos revealed no contribution to the e-pF by cells deriving from $\mathrm{Dbx}^{+}$progenitors. Moreover, immunostaining in Dbx $1^{\text {Lacz/LacZ }}$ embryos showed that the e-pF neurons as defined by Phox $2 \mathrm{~b}^{+}$, Isl $1,2^{-}$and vGlut2 $^{+}$expression ${ }^{2,3}$ were preserved (Supplementary Figure 2c,d).

\section{Impaired preBötC commissural connectivity.}

Apart from its rhythmogenic property, the other essential feature of the preBötC is its bilaterally synchronous mode of activity. We therefore explored whether this property was, like rhythmic activity, intrinsic to the Dbx1-derived population of neurons.

We first asked whether commissural connectivity in the preBötC was altered in Dbxi $1^{\text {LacZ/LacZ }}$ embryos. In Dbx $1^{\text {LacZ/+ }}$ preBötC slices, we recorded optically the responses evoked by electrical stimulation delivered between rhythmic spontaneous bursts by concentric bipolar electrodes positioned over the preBötC on one side or on the midline. In all cases $\left(n=3\right.$ slices and see ref. ${ }^{12,32}$ ), bilateral preBötC fluorescence changes were evoked (Fig. 5a,b), similar to those occurring spontaneously (data not shown) ${ }^{32}$. In contrast, in Dbx $1^{\text {Lacz/LacZ }}$ preparations $(n=4)$, preBötC stimulations evoked fluorescence changes spatially restricted to cells in the immediate vicinity of the stimulating electrode. Midline stimulations systematically failed to evoke any responses in the preBötC areas, or anywhere else in the slice (Fig. 5g,h). Thus, the Dbx1 null mutation disrupts all excitatory communication across the midline between ventrolateral areas of the medulla encompassing the preBötC. Corroborating this, in $\mathrm{Dbx} 1^{\text {laczl+ }}$ preBötC transverse slices, tracing experiments using unilateral injections of biocytin in the preBötC area, identified retrogradely-stained neurons in the contralateral preBötC as well as the presence of anterogradely-stained axonal terminals (Fig. 5c). Sixty seven percent of preBötC commissural interneurons retrogradely labeled with biocytin co-expressed B-Gal (Fig. 5d,j), $16 \%$ co-expressed Sst ${ }^{+}$ (Fig. 5e,k) and $8,5 \% \mathrm{Evx}^{+}$(Fig. 5f,I). In Dbx1 ${ }^{\text {LacZ/LacZ }}$ mutant preparations, the number of commissural preBötC interneurons was reduced by $89 \%$ largely owing to the loss of retrograde labeling in $\mathrm{B}-\mathrm{Gal}^{+}$cells (Fig. $\mathbf{5 i}, \mathbf{j}$ ). Collectively, these data show that preBötC commissural excitatory communication is

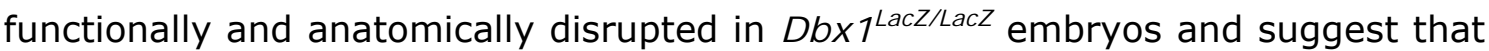
the formation of contralateral projections is an intrinsic property of Dbx1-derived cells. 


\section{Independent left/right rhythms in the absence of commissures.}

The Roundabout receptor 3 (Robo3) is a key regulator of commissure formation in the spinal cord and hindbrain ${ }^{16-18}$. Robo3 null (Robo3 ${ }^{\text {GFP/GFP }}$ ) mutants die at birth for still unknown reasons. We thus asked whether the preBötC bilateral synchronization was disrupted in these animals.

We first investigated whether Dbx1-derived cells of the preBötC express Robo3. In double heterozygous $\mathrm{Dbx} 1^{\mathrm{Lacz} /+} ; \mathrm{Robo}^{\mathrm{GFP} /+}$ embryos about $30 \%$ of $\mathrm{B}-\mathrm{Gal}^{+}$cells in the preBötC area were found to be GFP ${ }^{+}$, which corresponded to about half of $\mathrm{GFP}^{+}$cells present in the preBötC area (Supplementary Fig. 3a,b, Table $\mathbf{1}$ ). There was an absence of GFP expression in $\mathrm{B}-\mathrm{Gal}^{+}$cells in Dbx $1^{\text {Lacz/Lacz }}$, Robo3 ${ }^{\mathrm{GFP} /+}$ mutants (Supplementary Fig. 3), a finding consistent with disrupted commissural connectivity.

We then optically recorded activity of the preBötC in transverse slices $(n=6)$, or that of the facial motor nucleus concurrently with the electrical activity of C4 output in whole hindbrain preparations $(n=5)$ from E15.5 Robo3 ${ }^{\text {GFP/GFP }}$ embryos (Fig. 6). In slices, spontaneous rhythmic activity of the preBötC was preserved in the left and right preBötC, although with a slightly slower frequency than in Robo3 $^{\mathrm{GFP} /+}$ or wildtype embryos (Robo3 ${ }^{\mathrm{GFP} / \mathrm{GFP}}, f=3.1 \pm 0.2$ bursts/min, 12 preBötC from 6 slices; pooled controls, $4.5 \pm 0.4$ bursts/min, 26 slices). However, there was a complete loss of the bilateral synchronization (Fig. 6a). In whole hindbrain preparations, rhythmic co-active bursts of activity were recorded in the facial motor nucleus and $\mathrm{C} 4$ root on the same side of the midline but never from opposite sides ( $n=6$ preparations, Fig. 6d,e). Notably, the rhythmic activity in Robo3 ${ }^{\text {GFP/GFP }}$ preparations was suppressed by bath application of the preBötCdepressing $\mu$-opiate agonist DAMGO $(0.1 \mu \mathrm{M}, \mathrm{n}=3$, data not shown). Tracing experiments in which we injected biocytin unilaterally to the preBötC in Robo3 ${ }^{\text {GFP/GFP }}$ slice preparations revealed the absence of any midline-crossing axons (Fig. 6b,c). This confirms that Robo3 is required for axons to cross the midline in the hindbrain ${ }^{16}$. Accordingly, Robo3 ${ }^{\text {GFP/GFP }}$ E18.5 embryos $(n=5)$ delivered from uterine horns or PO neonates $(n=2)$ featured a conspicuous split breathing behavior characterized by independent left and right rhythmic contractions of the diaphragm (see Supplementary Movie 1) indicating that, in the absence of Robo3, unilateral rhythmic motor circuits can be maintained throughout the hindbrain and the spinal cord. Such uncoordinated left and right rhythmic respiratory efforts probably preclude efficient breathing thus explaining cyanosis and death of all (7/7) E18.5-delivered or born Robo3 ${ }^{\text {GFP/GFP }}$ animals within 8 hours of birth. These data are consistent with the essential role of Robo3 signaling for commissural axon pathfinding in the hindbrain and also suggest that commissural connectivity may be dispensable for emergence of respiratory rhythmic activity.

Finally, we asked whether Robo3-mediated signaling was required cellautonomously in Dbx1-derived neurons for bilateral synchrony of the preBötC using a mouse carrying a Robo3 conditional allele (Robo3 $\left.{ }^{\text {lox/lox }}\right)^{17}$. PreBötC rhythm generation and bilateral synchronization was compared between wildtype (Fig. 7 a-d) and Dbx1:: Cre; Robo3 ${ }^{\text {lox/lox }}$ mutant mice (Fig. 7e-h). As previously observed 
in Robo3 ${ }^{\text {GFP/GFP }}$ mutants, in all transverse slice preparations tested $(n=6)$, the Robo3 conditional mutation spared rhythmic activity in left and right preBötC (4.9 \pm 0.4 bursts/min, 12 preBötC), but led to their complete de-synchronization (Fig. 7e,f). In addition, biocytin tracing experiments in Dbx1::Cre; Robo3 ${ }^{\text {lox/lox }}$ preparations showed a $92 \%$ deficit of stained commissural interneurons (Fig.

7g-h; wildtype : $56.4 \pm 5.3$ cells/preBötC, $n=5$; Dbx1::Cre; Robo3 ${ }^{\text {lox/lox }}: 4.3 \pm$

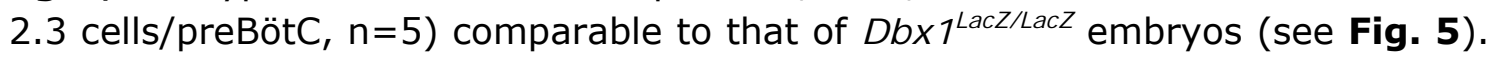
Like Robo3 ${ }^{\text {GFP/GFP }}$ mutants, none of Dbx1::Cre;Robo3 ${ }^{\text {lox/lox }}$ animals (this will be described in a separate report) survived after birth.

These data demonstrate the necessary role of Dbx1-derived neurons in enforcing bilateral synchrony of the preBötC, an essential feature that depends on the 30 $\%$ fraction of Dbx1-derived cells in the complex that express Robo3. Thus, like rhythm generation, bilateral synchrony of the pre-BötC emerges as a property intrinsic to Dbx1-derived cells.

\section{DISCUSSION}

Using molecular genetic strategies in embryos, we found that Dbx1expressing progenitors give rise to the neurons necessary for rhythm generation and bilateral synchrony of the preBötC, the main respiratory oscillator. Spontaneous, collective and bilateral preBötC rhythmic behavior is permanently abrogated in the absence of Dbx1, leading to the complete absence of respiratory efforts at birth. The $\mathrm{Dbx} 1$ null mutation causes a massive reduction of the number of glutamatergic preBötC interneurons including its constitutive NK1R- and Sst-expressing neurons. The glutamatergic neurons born outside the ventral domain of $\mathrm{Dbx}^{+}$progenitors, including those of the spared e-pF oscillator, are unable to compensate for the lethal respiratory deficit resulting from the Dbx1 inactivation. In addition, preventing commissural Dbx1-derived interneurons from projecting axons across the midline is sufficient to desynchronize the rhythms of left and right preBötC. Thus, in the hindbrain, the homeobox gene Dbx1 stands upstream of a developmental program providing neurons with the molecular properties that ensure built-in rhythm generation, bilateral synchrony, as well as to some extent, neuromodulatory influence and responsiveness of the vital preBötC respiratory oscillator.

Rhythm generation in the preBötC relies on glutamatergic synaptic transmission. In vitro, the preBötC rhythmic activity can be reversibly suppressed by the transient inhibition of AMPA/Kainate receptors ${ }^{5,32,34}$ and its embryonic emergence is prevented in Vglut2 null mutants ${ }^{12}$. The deletion ${ }^{9,} 35$ or the silencing ${ }^{10}$ respectively of NK1R- and Sst-expressing preBötC neurons disrupts breathing in the adult rat. We have shown that NK1R- and Sstexpressing preBötC interneurons are lacking in $\mathrm{Dbx} 1^{\text {Lacz/LacZ }}$ mutants. Since $\mathrm{NK}_{1 \mathrm{R}}{ }^{+}$and $\mathrm{Sst}^{+}$neurons may form only partially overlapping neuronal populations in the preBötC ${ }^{10}$, the severe respiratory impact of their deletion is probably due to their role in supporting the glutamatergic neurotransmission required for rhythm generation ${ }^{7,}{ }^{36}$. Indeed, most ( $82 \%$ ) preBötC Dbx1-derived 


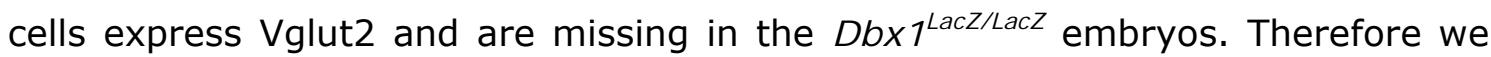
propose that the complete absence of rhythm in the preBötC of $\mathrm{Dbx} 1^{\text {Lacz/LacZ }}$ embryos results from a depletion of glutamatergic interneurons. This is consistent with current models of rhythm generation in the preBötC in which glutamatergic interneurons contribute the recurrent excitatory connections, priming the collective and periodic all or none population burst firing defining the oscillator activity ${ }^{1,5,7,12,34,36 .}$

The associated collective loss of NK1R, Sst and chiefly Vglut2 expression demonstrate that $\mathrm{Dbx} 1$ is required to specify the molecular properties that characterize the preBötC anatomically ${ }^{8}, 9$ and underlie its function. The $\mathrm{Dbx} 1^{\text {Lacz/LacZ }}$ anatomical and functional preBötC phenotypes are not recapitulated in a Pax7::Cre; Dbx $1^{\text {DTA }}$ mice, indicating that little if any of the phenotype is contributed by cells originating in the dorsal domain of $\mathrm{Dbx} 1^{+}$progenitors. Therefore, the critical neurons ensuring rhythm generation of the preBötC belong to the VOv interneuronal class ${ }^{24}$.

A propensity shared by both preBötC cells and V0 spinal cord interneurons ${ }^{24}$ is to project axons across the midline. We have identified Robo 3 as a necessary receptor for this process. Notably, in Dbx1::Cre;Robo3 ${ }^{\text {lox/lox }}$ embryos, left/right synchrony of the preBötC is abolished. However, rhythmic preBötC are spared on either side of the midline in conditional or straight Robo3 null mutants. This is consistent with previous observations of maintained preBötC rhythmic activity in slices following midline section ${ }^{37}$ or in island preparations ${ }^{38}$ thus demonstrating that commissural connectivity is dispensable for both emergence and maintenance of the rhythm. Therefore, rhythm generation appears as a Dbx1-dependent and Robo3-dispensable process critically relying on ipsilateral glutamatergic re-excitatory connections.

The strength of the link between Dbx1 and Robo3 probably ensures that the preBötC constitutes a major commissural hub of central respiratory circuits. The adult respiratory motor command in vivo involves sequential phases (e.g. inspiratory, post-inspiratory and expiratory ${ }^{1,}{ }^{39}$ ), that proceed in synchrony across the midline. Robustness of bilateral co-activity of the central control of breathing rests on the existence of multiple excitatory commissural relays at sensory integrative and pre-motor levels ${ }^{39}$ that all probably depend on Robo3. A major finding of the present study is that a large fraction of preBötC cells endowed with both glutamatergic and commissural identities are Dbx1dependent. This includes $\mathrm{VOV} \mathrm{Sst}{ }^{+}, \mathrm{Evx}^{+}$and probably the majority of $\mathrm{NK}^{+} \mathrm{R}^{+}$ cells collectively candidate neurons for rhythm generation and bilateral synchrony. Therefore, our data demonstrate that ventral $\mathrm{Dbx} 1^{+}$progenitors of the caudal hindbrain are fated to become confined sources of rhythmic neural activity with built-in synchronicity across the midline. This is consistent with previous findings in the adult rat showing that $\mathrm{Sst}^{+}$neurons contribute to the preBötC commissural apparatus ${ }^{7,8}$ and specifically project onto major respiratory 
relay structures in the brainstem including the preBötC ${ }^{7}{ }^{8}$. Spinal Evx1 cells comprise rostrally projecting commissural interneurons ${ }^{40}$ that may, in the hindbrain, contribute to couple the preBötC with the e-pF ${ }^{2}$. Future experiments should reveal to what extent Dbx1-derived and/or Robo3-expressing neurons further support the connectivity of the respiratory rhythm generating network, such as between the preBötC and the e-pF or between the oscillator level and pre-motor circuits. In any case, Dbx1 and Robo3 stand as major determinants of the respiratory central network.

Inactivation of Robo3 has been shown to spare the ability of axons to navigate and finally project onto their normal target, but on the ipsilateral side ${ }^{17}$. Consistent with this, the rhythmic entrainment of facial and C4/phrenic motor neuronal targets was spared unilaterally in Robo3 null mutants. Therefore, at the preBötC level, re-routed axons probably form ipsilateral connections with other preBötC neurons that are, in terms of rhythm generation, functionally equivalent to those normally established with contralateral partners. Robo3 expression in Dbx1-derived cells is lost in the absence of Dbx1 protein. Previous studies have identified several transcription factors that directly control the expression of Robo receptors $^{41,42}$. In Lhx2/Lhx9 knockout, the selective loss of Robo3 expression on a subset of DI1 spinal cord commissural interneurons prevents their axons from crossing the midline. Our results suggest that Robo3 could also be a transcriptional target of Dbx1.

We are not aware of any human respiratory deficits that would point to an unbalanced left/right central respiratory command. Recent studies have shown that mutations in genes encoding axon guidance receptors such as $\mathrm{DCC}^{43}$ and Robo $3^{15}$ lead to specific motor deficits that, at least to some extent, involve hindbrain commissures. The rare human syndrome horizontal gaze palsy with progressive scoliosis (HGPPS) is caused by mutations in the Robo3 gene. Patients are unable to perform conjugate lateral eye movements and have reduced hindbrain and spinal cord commissures, including uncrossed corticospinal tract and dorsal column-medial lemniscus ${ }^{15}$. One of the two signature traits of HGPPS patients is a severe scoliosis of unknown origin. Although speculative, the dramatic independence of left/right breathing activities and diaphragm contractions in Robo3 null mice raises the possibility that, owing to its continuous character, even a mild inbalance of the periodic mechanical constraint exerted by respiration on the axial skeleton may result in the abnormal posture of HGPPS patients.

Hindbrain interneurons in para-facial and para-vagal locations organize into rhythmically active cellular assemblies in the mouse embryo and pre-figure respectively the two oscillators comprising the fetal and postnatal respiratory rhythm generating network. Several features indicate that the e-pF and PreBötC oscillators are not serially homologous structures. They derive from respectively dorsal and ventral domains of the neural tube, giving rise to $\mathrm{Db} 2^{3}$ and $\mathrm{V} 0$ neurons. In mice, their constituent cells are respectively born before E10.5 (JF 
Brunet and C Goridis personal communication) and during E10.5-11.5 (this study), a sequence matching their later functional emergence at $E 14.5^{2}$ and E15.5 ${ }^{32}$. We have previously shown that the preBötC oscillator could emerge in the absence of e-pF oscillator ${ }^{2}$ and show here that the converse is also possible in the $\mathrm{Dbx} 1^{\mathrm{Lac} / \mathrm{LacZ}}$ mutant. This firmly establishes that the embryonic parafacial and the preBötC oscillators arise independently. Thus, the preBötC appears to be inserted within an e-pF containing network; the complete absence of breathing in Dbx $1^{\text {Lacz/Lacz }}$ mutants in the presence of an active e-pF oscillator further demonstrates that the preBötC then takes on a prominent role.

Knowledge about the intrinsic logic of central pattern generating neuronal networks (CPGs) remains a challenging issue in neuroscience. The respiratory rhythm generating network in the hindbrain controls an exclusively synchronous bilateral motor behavior ${ }^{1}$, while the locomotor CPG in the spinal cord, in most vertebrates, is able to transit between left/right alternating and synchronous modes ${ }^{44}$. Our study points to the allocation of a pool of neural progenitors for the assembly of these two CPGs, and to Dbx1 and Robo3, expressed in these cells, as candidate targets of regulatory interaction that may constrain their respective operating principles. Dbx1-derived V0 commissural interneurons in the lumbar spinal cord have been shown to have a largely (70 \%) inhibitory glycinergic or GABAergic nature while the remainder express Vglut ${ }^{24}$. The absence of v0 interneurons in Dbx $1^{\text {Lacz/LacZ }}$ mutants leads, at the spinal cord level, to a marked increase in incidence of abnormal bilateral co-bursting of the left and right flexor/extensor motoneurons during drug-induced locomotion in vitro ${ }^{24}$. Thus, at the spinal level, the vo interneurons mediate crossed inhibition conditioning left/right alternating phasic activity. In the hindbrain, three independent lines of evidence - electrical stimulation, retrograde tracing and Robo3 conditional deletion - demonstrate the commissural phenotype of Dbx1-derived interneurons. However, unlike in the spinal cord, $82 \%$ of V0 interneurons in the preBötC area are glutamatergic and therefore directly transmit their excitation across the midline to enforce bilateral phasing of activities. Recently glycinergic neurons, estimated to contribute to $20 \%$ of the preBötC population, were shown to have pacemaker membrane properties ${ }^{45}$. Although neither the Dbx1-derived nature of these cells nor altogether the pacemaker status of Dbx1-derived cells have been established, the possibility exists that these glycinergic cells could complement the fraction of Dbx1-derived glutamatergic neurons. If this were the case, our results suggest that the different balance between the excitatory and inhibitory $\mathrm{V} 0$ interneurons in the hindbrain and the spinal cord may be a fundamental divergence of the respiratory and locomotor CPGs' building blocks. Regulatory genes specifying the glutamatergic fate in the preBötC are unknown but differ from those operating in the dorsal neural tube, which require $T / \times 3^{46}$, a transcription factor not expressed in the Vo domain. Because an optimal excitatory/inhibitory ratio would appear to take on a crucial importance, an immediate concern is now to investigate regional regulatory mechanisms 
affecting PO progenitors and the ensuing neurogenic programs that control the excitatory versus inhibitory cell fate of VOv interneurons in the caudal hindbrain.

\section{ACKNOWLEDGEMENTS}

We thank J-F. Brunet, C. Goridis and A. Lumsden for comments on the manuscript, Sonia Karaz for providing specimen and Sandra Autran and Valérie Mézières for technical assistance with genotyping. J.B. is supported by Région Ile-de-France and the Fondation pour la Recherche Médicale (FRM). This work was supported by grants from FRM Equipe FRM grant to A.P., the Association Française contre les Myopathies (AFM, ASS-SUB06-00123) to A.C., the Ville de Paris (2006 ASES 102) to A.P., the Agence Nationale de la Recherche (ANR-05NEUR-007-01 BIS) to A.P., (ANR-08-MNPS-030-01) to A.C., (ANR-07-NEUR-00701 ) to G.F. This work benefited from the facilities and expertise of the Imagif Cell Biology Unit and the Anicampus mouse facility of the Gif-sur-Yvette campus. This work was supported by Centre National de la Recherche Scientifique and the Institut de la Santé et de la Recherche Médicale.

\section{AUTHOR CONTRIBUTIONS}

J.E., A.P., J.C. and G.F. conceived the study, N.R. and A.C. designed Robo3 experiments, J.B. and M.T-B. performed the experiments, V.D. performed vGlut2 ISH, J.B., M.T-B. and G.F. analysed the data, J.E., A.C. and A.P. provided reagents and mice, G.F. wrote the paper. All authors discussed the results and implications and commented on the manuscript at all stages.

\section{LEGENDS}

Figure 1 Disrupted breathing and rhythm generation in the preBötC of Dbx1 null mice.

$(\mathbf{a}, \mathbf{b})$ Plethysmographic recordings of the ventilation of $\operatorname{Dbx} 1^{\text {LacZ/+ }}$ (a) and Dbxi $1^{\text {LacZ/Lacz }}$ (b) E18.5 embryos 1 min after surgical delivery. All Dbx1 $1^{\text {LacZ/+ }}$ initiated respiratory cycles of inspirations (upward deflections) and expirations (downward deflections), whereas all Dbx $1^{\text {LacZ/LacZ }}$ mutants animals did not show any sign of ventilation and died cyanotic. $(\mathbf{c}, \mathbf{d})$ Bilateral rhythmic fluorescence changes $(\Delta F / F)$ of the preBötC in E15.5 slice preparations (c, dorsal at top) were absent in the mutant (d). (e,f) Corresponding concurrent electrophysiological (Int. top traces) and optical $(\Delta F / F$, bottom traces) recordings showing spontaneous preBötC rhythmic bursts increasing in frequency in the presence of substance $\mathrm{P}$ (SubP) in Dbx $1^{\text {LacZ/+ }}$ preparations (e) ; activity of the preBötC was absent in $\mathrm{Dbx} \mathbf{1}^{\text {Lacz/LacZ }}$ preparations and could not be induced by $\operatorname{SubP}(\mathbf{f}) .(\mathbf{g}, \mathbf{h})$ Calcium imaging of the ventral surface in $\mathrm{E} 15.5 \mathrm{Dbx} 1^{\mathrm{LacZ} /+}(\mathbf{g})$ and $\mathrm{Dbx} 1^{\text {LacZ/LacZ }}$ (h) whole hindbrain preparations. Rhythmic activity of the e-pF oscillator (green outline), partially coupled at this stage to that of nVII (white dotted outline) is maintained in the Dbx1 null mutant. $(\mathbf{i}, \mathbf{j})$ Simultaneous optical recordings (top two traces) of the e-pF (green) and of the nVII (black) and electrophysiological recording of the fourth cervical spinal root (C4, bottom trace) in DbxilacZ/+ (i) and $\operatorname{Dbx} 1^{\text {LacZ/LacZ }}(\mathbf{j})$ preparations. The rhythmic activity of the e-pF is spared in 
Dbx $1^{\text {LacZ/LacZ }}$ preparations albeit in the absence of any activity of the motor outputs. Scale bars (in $\mu \mathrm{m}$ ): VII: facial motor nucleus.

Figure 2 Dbx1-derived cells in the preBötC are rhythmically-active.

(a) Expression of Dbx1 protein in a $\mathrm{Dbx} 1^{\mathrm{LacZ} /+} \mathrm{E} 10.5$ transverse hindbrain section.

(b) B-Gal protein expression. (c) B-Gal protein expression (green) in half of a E15.5 transverse section (midline at right) and Islet1,2 (red) expressed by the XIIn and the n.a. (d) Peak fluorescence change $(\Delta F / F)$ during one burst of the preBötC in half of a physiological slice (midline at left). (e) Expression of Dbx1 in a Dbxi ${ }^{\text {iresGFP }}$ E10.5 transverse hindbrain section. (f) GFP protein expression. (g) GFP expression in a E15.5 preBötC slice preparation during an electrophysiological recording session. (h) Same field (blue rectangle in $\mathbf{g}$ ) showing GFP expression (red) and Calcium Green-1 AM loaded cells (CaG, green) and at higher magnification the merged image (right) used to derive the individual rhythmic activity of 11 double labeled (yellow) preBötC cells. (i) Corresponding fluorescence changes of individual cells (black) and averaged preBötC signal (blue). (j) GFP expression (green) and DIC image used to position the patch micropipette (arrows). (k) Membrane potential changes (top trace) of a $\mathrm{GFP}^{+}$preBötC neuron showing spontaneous rhythmic burst discharges of action potential phased to the integrated (Int. bottom trace) preBötC population bursting activity. (I) Membrane current changes in the same neuron showing rhythmic bursting volleys of synaptic currents phased to the activity of the preBötC. Scale bars (in $\mu \mathrm{m})$ : a,b,e,f (100); c,d (500); h (50); j (15). E10.5 sections are taken from the level of rhombomere 7. n.a.: nucleus ambiguus, XII: hypoglossal nucleus.

Figure 3. Phenotypic profiles of Dbx1-derived cells of the preBötC. Immunohistochemical stainings in $\operatorname{Dbx} 1^{\text {LacZ/+ }}(\mathbf{a}-\mathbf{j})$ and $\mathrm{Dbx} 1^{\text {LacZ/LacZ }}$ (k-s) embryos. (a,k) B-Gal (green) and Islet1,2 (red) expressions in E15.5 transverse sections. (b,I) Sst expression, (c, $\mathbf{m})$ NK1R expression at E18.5. (d, $\mathbf{n})$ Sst expression in the preBötC area (dotted square in $\mathbf{a}, \mathbf{k}) .(\mathbf{e}, \mathbf{0})$ Merged Islet1,2 (blue), B-Gal (green) and Sst (red) expressions, insert showing a co-labeled B$\mathrm{Gal}^{+}, \mathrm{Sst}^{+}$neuron. (f, p) NK1R expression, $(\mathbf{g}, \mathbf{q})$ merged Islet1,2 (blue), B-Gal (green) and NK1R (red) expressions, insert showing a co-labeled $B-\mathrm{Gal}^{+}, \mathrm{NK} \mathrm{R}^{+}$ neuron. (h,r) Vglut2 ISH, (i,s) merged expressions of $B-G a l$ (green) immunoreactivity combined to Vglut2 ISH (red). (j) Vglut2 ISH (red) and immunostaining for Sst (green). (t) Histogram showing the fractions of Sst ${ }^{+}$cells in the preBötC area that are co-expressing B-Gal (white) or not (gray) in both genotypes. Note that Sst expression relies on Dbx1-derived neurons and is suppressed in the Dbx1 null mutant. (u) Same histogram for Vglut2 ${ }^{+}$cells. About half of Vglut2 expression in the preBötC area is accounted by Dbx1-derived cells (white) and is largely absent in the Dbx1 null mutant. In histograms, neurons were counted on 3-8 sections from $5\left(\mathbf{t}, \mathrm{Dbx} 1^{\text {LacZ/+ }}\right)$ and $4\left(\mathbf{t}, \mathrm{Dbx} 1^{\text {LacZ/LacZ }} ; \mathbf{u}\right)$ animals, and results are given as the average number of cells in the preBötC 
area per $20 \mu \mathrm{m}$ section; error bars represent s.e.m. Scale bars (in $\mu \mathrm{m}$ ): a-c, km (300); d-i, n-s (50), insets (15); j (25). n.a.: nucleus ambiguus.

Figure 4 The preBötC derives from ventral $\mathrm{Dbx} 1^{+}$progenitors.

(a) Pax3/7 (blue), Dbx1 (green) and Evx1 (red) expressions in a E10.5 Dbx $1^{\text {LacZ/+ }}$ hindbrain transverse section. Right panel: magnification of the square in the left image.Evx $1^{+} \mathrm{V}_{\mathrm{V}} \mathrm{v}$ interneurons arise laterally from the ventral $\mathrm{Dbx} 1^{+}, \mathrm{Pax} 7^{-}$ progenitors and not from dorsal $\mathrm{Pax} 7^{+}, \mathrm{Db} \times 1^{+}$progenitors giving rise to $\mathrm{V} 0_{\mathrm{D}}$ interneurons. $(\mathbf{b}, \mathbf{c})$ Same experiments in Dbx1 $1^{\text {LacZ/LacZ }}$ sections $(\mathbf{b})$, showing the complete loss of $\mathrm{Dbx} 1^{+}$progenitors, and from the Pax7::Cre; Dbx $1^{\text {DTA }}$ mutant (c) showing Dbx1 protein expression restricted to the ventral domain. (d) Calcium imaging in a Pax7::Cre; Dbx1 ${ }^{\text {DTA }}$ E15.5 slice showing preserved preBötC rhythmic activity. (e-g). E18.5 transverse sections in the Pax7::Cre;Dbx1 ${ }^{\text {DTA }}$ mutant showing in the preBötC area the conserved expressions of Sst (e), Vglut2 (f) and $\operatorname{NK1R}(\mathbf{g})$. (h,i) Expressions of Islet1,2 (blue), B-Gal (green) and Evx1 (red) in $\operatorname{Dbx} 1^{\text {LacZ/+ }}(\mathbf{h})$ and $\operatorname{Dbx} 1^{\text {LacZ/LacZ }}(\mathbf{i})$ in the preBötC area at E15.5 showing two populations of Evx1 $1^{+}$cells co-expressing (yellow) or not(red) B-Gal. Note the selective lack of $\mathrm{B}-\mathrm{Gal}^{+}, \mathrm{Evx} 1^{+}$cells in Dbx1 ${ }^{\text {LacZ/LacZ }}$ mutants. (j) Evx $1^{+}$cells are preserved in the Pax7::Cre; Dbx $1^{\text {DTA }}$ mutant. (k) Histogram (mean \pm s.e.m.) showing the changes, in the preBötC area, in the number of Evx1 $1^{+}$cells and, when applicable, the fractions co-expressing B-Gal (yellow) or not (red). (I-o) BGal (blue, I), Evx1 (green, m), Vglut2 (red, n) and merged expressions (o)in the preBötC area. Note the combined expressions of $B-G a l$ and $\mathrm{Vglut2}$ in all Evx1 ${ }^{+}$ cells (arrows). Scale bars (in $\mu \mathrm{m})$ : a (200); inset, b, c (50); d (500); e-j (100); I-o (50). E10.5 sections are taken from the level of rhombomere 7. n.a.: nucleus ambiguus.

Figure 5 preBötC commissural connectivity is disrupted in Dbx1 null mutants. preBötC commissural connectivity in Dbx1 $1^{\text {LacZ/+ }}(\mathbf{a}-\mathbf{f})$ and Dbx1 $1^{\text {LacZ/LacZ }}$ (g-i) E15.5 slices. $(\mathbf{a}, \mathbf{b}, \mathbf{g}, \mathbf{h})$ Calcium Green-1 AM loaded preBötC slices used to optically record the left (black outlined region) and right (red outlined region) preBötC evoked responses $(\Delta F / F$, middle panel) showing the location of the stimulating electrode on the right preBötC $(\mathbf{a}, \mathbf{g})$ or on the midline $(\mathbf{b}, \mathbf{h})$. Color traces at right are super-imposed responses of the right (red) and the left (black) preBötC to five individual electrical stimulations (black arrowhead). Note the absence of contralateral responses in the Dbx1 null mutant. (c,i) Biocytin (green) and NK1R (red) expressions in $\mathrm{E} 15.5$ slices injected with biocytin onto the right preBötC (arrow). Right panels are higher magnifications of the contralateral preBötC (dotted square) showing abundant retrogradely-labeled somas (green) in Dbxi $1^{\text {LacZ/+ }}$ (c) but not in Dbxi $1^{\text {LacZ/LacZ }}$ (i) slices. (d-f) Biocytin-labeled somas (green) in the preBötC area of $\mathrm{Dbx} 1^{\mathrm{LacZ} /+}$ slices, counterstained for (red) B-Gal (d), Sst (e) and Evx1 (f). (j) Histogram showing the fractions of biocytin-labeled preBötC cells co-expressing B-Gal (white) or not (gray) in both genotypes. Note the dramatic reduction of commissural neurons in null mutants, owing to the absence of double-labeled cells. ( $\mathbf{k}, \mathbf{I})$ Histograms showing, in Dbx $1^{\text {LacZl+ }}$ slices, 
the fractions of biocytin-labeled cells co-expressing (white) Sst (k) and Evx1 (I). Results are given as the average number of biocytin ${ }^{+}$cells in the preBötC area co-expressing the marker per physiological slice; error bars represent s.e.m. Scale bars (in $\mu \mathrm{m})$ : $\mathbf{a}, \mathbf{b}, \mathbf{g}, \mathbf{h}(500) ; \mathbf{c}, \mathbf{i}(500)$; insets (50); d-f (20). n.a.: nucleus ambiguus.

Figure 6 Left/right de-synchronization of the preBötC and of motor neuronal outputs in Robo3 ${ }^{\text {GFP/GFP }}$ embryos.

(a) Calcium Green-1 AM loaded E15.5 preBötC slice used to optically record activities of the left ( $L$ outlined region) and right ( $R$ outlined region) preBötC ( $L$ and $\mathrm{R}$ traces below). Note the presence of spared rhythmic calcium fluorescence changes in both the left and right preBötC ( $\Delta F / F$ panels sampled at the time indicated by asterisks on traces) in the absence of bilateral synchronization. (b) Biocytin injections (white arrow) in wildtype (WT, top) and Robo $3^{\text {GFP/GFP }}$ (bottom) physiological slices showing respectively the presence (black arrowhead) and the absence of contralaterally-labeled preBötC neurons. (c) Higher magnification view of NK1R (red) and Biocytin (green) expressions over the midline in the same slices, showing that midline crossing axons present in WT (left) are missing in Robo3 ${ }^{\text {GF/GFP }}$ mutant slices. (d) Whole hinbrain E15.5 preparations (left schematic, anterior at top) used to concurrently record, optically the activities of the left and right facial motor nucleus (nVII) anteriorly, and electrophysiologically the left and right $\mathrm{C} 4$ spinal root, posteriorly. Note on corresponding traces that the rhythmic activity of the $\mathrm{nVII}(\Delta \mathrm{F} / \mathrm{F}$ panels, midline: dotted line) and $\mathrm{C} 4$ motor outputs is bilaterally-synchronized in a wildtype preparation (WT, top set of traces) but unilaterally synchronized in a Robo3 ${ }^{\text {GFP/GFP }}$ preparation (bottom set of traces). (e) Corresponding left/right nVII cross-correlations for WT (dotted

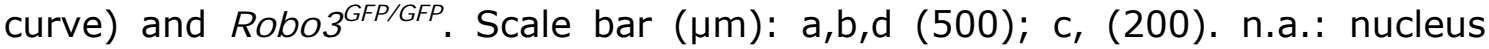
ambiguus.

Figure 7 Left/right de-synchronization of the preBötC in the Dbx1:: Cre; Robo $3^{\text {lox/lox }}$ conditional mutant.

(a) Integrated population electrical activity of the left ( $L$ Int.) and right ( $R$ Int.) preBötC recorded in a E15.5 wildtype slice showing rhythmic and synchronized bursts. (b) Corresponding left/right preBötC cross-correlogram. (c) Islet1,2 (blue), Biocytin (green) and NK1R (red) expressions in a physiological slice injected with biocytin onto the right preBötC (arrow) showing retrogradely-filled contralateral preBötC neurons (dotted square) shown at higher magnification in (d). (e-f) Same experiments as in (a) and (b) in a Dbx1::Cre; Robo3 $3^{\text {lox/lox }}$ conditional mutant physiological slice showing de-synchronized rhythmic activities of the left and right preBötC (e), resulting in a flat cross-correlogram (f). (g-h) Same experiments as in (c) and (d) in the Dbx1::Cre; Robo3 ${ }^{\text {lox/lox }}$ mutant showing that prebötC commissural connectivity is disrupted and that the number of retrogradely-labeled contralateral preBötC neurons is massively reduced $(\mathbf{h})$. Scale bars (in $\mu \mathrm{m}): \mathbf{c}, \mathbf{g}(300) ; \mathbf{d}, \mathbf{h}(50)$. n.a.: nucleus ambiguus. 
Supplementary Figure 1 Dbx1-derived preBötC cells are born during the E10.5-11.5 period.

(a-c) Immunohistochemical stainings showing the perdurance of $B-G a l$ protein between E15.5 and E18.5. (a,b) Merged expressions of Islet1,2 (red) and B-Gal (green) on $20 \mu \mathrm{m}$ transverse sections of Dbx1 ${ }^{\mathrm{LacZ} /+}$ embryos at E15.5 (a) and E18.5 (b). Half-sections are shown, with the dotted line representing the midline and the dotted square the preBötC area. (c) Histogram showing, in the preBötC area, the average number ( \pm s.e.m.) of $B-\mathrm{Gal}^{+}$cells per $20 \mu \mathrm{m}$ section. There is no obvious difference between the two developmental stages. (d-i) Birthdating experiments of $\mathrm{B}_{-\mathrm{Gal}^{+}}$cells in the preBötC area on $\mathrm{Dbx} 1^{\mathrm{LacZ} /+}$ embryos. (d-h) Immunohistochemical stainings on $20 \mu \mathrm{m}$ transverse sections at E15.5 showing BrdU (red), B-Gal (green) and merged expressions (right panels) after single pulse BrdU injection (inj.) at indicated stage. Co-expression (yellow) was only observed when BrdU was injected at E10.5 (e) or E11.5 (f). (i) Histogram

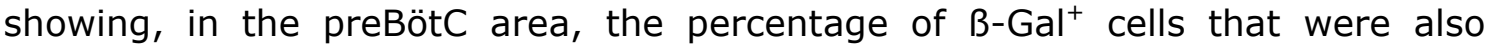
positive for BrdU, for each stage of BrdU injection (BrdU inj.). Scale bars (in $\mu \mathrm{m})$ : a,b (300); d-h (50). n.a.: nucleus ambiguus.

Supplementary Figure $2 \mathrm{Phox}_{2 \mathrm{~b}^{+}}$and $\mathrm{Vglut}^{+}$cells of the e-pF oscillator are maintained in Dbxl null mice.

Immunohistochemical stainings and ISH at E15.5 on $20 \mu \mathrm{m}$ transverse sections

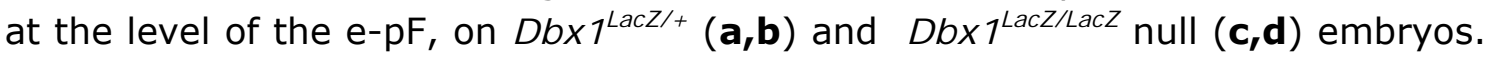
(a) Merged expressions of Islet1,2 (blue), B-Gal (Green) and Phox2b (red) showing Phox $2 b^{+}$,Islet $1,2^{-}$e-pF cells (arrows) located beneath and lateral to the facial motor nucleus (nVII, expressing both markers; left sides are shown). Note that none are co-expressing B-Gal. (b) Merged expressions of Islet1,2 (blue), BGal (Green) and vGlut2 ISH (red), showing that glutamatergic e-pF cells (arrows) are not co-expressing $B-G a l .(\mathbf{c}, \mathbf{d})$ Same experiments as in (a) and (b) in $\mathrm{Dbx} 1^{\text {LacZ/LacZ }}$ null embryos, showing that e-pF cells are maintained (arrows). Scale bar (in $\mu \mathrm{m}): 200$.

Supplementary Figure 3 Robo3 is expressed by Dbx1-derived interneurons. Immunohistochemical stainings of $\mathrm{E} 15.5$ transverse sections of Robo $3^{\mathrm{GFP} /+}$ embryos also carrying either the Dbx1 heterozygous mutation $\left(\mathrm{Dbx} 1^{\mathrm{LacZ} /+} ; \mathrm{Robo3}^{\mathrm{GFP} /+}, \mathbf{a , b}\right)$ or the Dbx1 null mutation (Dbx $1^{\text {LacZ/LacZ; Robo3 }}{ }^{\mathrm{GFP} /+}$, c,d). (a) GFP expression (Green), B-Gal expression (red) and merged expressions in the preBötC area showing co-labeled (yellow) somas. (b) Histogram showing, in the preBötC area, the fractions of $\mathrm{GFP}^{+}$cells, presumably commissural, that are Dbx1-derived (yellow) or not (green). (c) Same experiment as in (a) on a Dbx1 null embryo. Note the almost complete absence of double-labeled somas. (d) Same quantification as in (b) in a Dbx1 null embryo, showing a reduction of the total number of $\mathrm{GFP}^{+}$cells, largely owing to the lack of double-labeled $\mathrm{GFP}^{+},{ }, \mathrm{Bal}^{+}$cells. In (b) and (d), cells were counted in the preBötC area in 2-7 $20 \mu \mathrm{m}$ sections from 3 and 2 animals respectively and 
results are given as the average number of cells in the preBötC area per $20 \mu \mathrm{m}$ section ( \pm s.e.m.). Scale bars (in $\mu \mathrm{m}$ ): 50. n.a.: nucleus ambiguus

Supplementary Video1 Split left/right breathing in Robo3 mutants.

The movie shows a $7 \mathrm{~s}$ sequence of spontaneous breathing of an E18.5 Robo3 ${ }^{\text {GFP/GFP }}$ fetus shot 15 min after surgical delivery, and 5 hours before death. The animal is exposed ventral side up and features left/right de-synchronized movements of the rib cage.

\section{Table 1. Summary of cellular phenotypes in Dbx $1^{\text {LacZ }+}$ and Dbx $1^{\text {LacZ/ Lacz }}$ embryos}

${ }^{a}$ In parenthesis, ratio of the total number of cells counted in 3 to 9 sections from n animals.

$\%$ of $\beta-\mathrm{Gal}^{+}$cells expressing the marker

$\operatorname{Dbx} 1^{\text {LacZl+ }}$

$\operatorname{Dbx} 1^{\text {LacZ/LacZ }}$

$\operatorname{Dbx} 1^{\text {LacZl+ }}$

$\operatorname{Dbx} 1^{\text {LacZ/LacZ }}$

Marker

$\begin{array}{lllll}\text { Vglut2 } & 81,7(917 / 1122, n=4)^{a} & 8,4(198 / 2351, n=4) & 52,0(917 / 1765, n=4) & 9,6(198 / 2073, n=4) \\ \begin{array}{l}\text { Robo3 } \\ \text { (GFP) }\end{array} & 28,5(441 / 1547, n=3) & 4,2(33 / 784, n=2) & 44,7(441 / 986, n=3) & 8,3(33 / 398, n=2) \\ \text { Evx1 } & 31,3(471 / 1505, n=3) & 0,4(7 / 1973, n=3) & 53,9(471 / 874, n=3) & 1,4(7 / 494), n=3) \\ & 13,2(254 / 1921, n=5) & 0,0(1 / 2351, n=4) & 91,1(254 / 284, n=5) & 0,0(1 / 10, n=4)\end{array}$

Left: proportions of Dbx1-derived cells expressing the marker, right: proportions of cells expressing the marker that are Dbx1-derived in the two genotypes.

\section{METHODS}

Experiments were performed in keeping with European and French agricultural ministry guidelines for the care and use of laboratory animals (Council directives 2889 and 86 / 609 / EEC).

\section{Animal strains}

All mutant mice lines were described previously: Dbx $1^{\text {nlsLacz }} 29$ (Dbx $\left.1^{\text {Lacz }}\right)$, Dbx1::Cre ${ }^{47}$, Robo3 ${ }^{\text {GFP }}{ }^{18}$, Robo3 ${ }^{\text {lox }}{ }^{17}$, Pax7::Cre ${ }^{48}$. Dbx1 ${ }^{\text {iresGFP }}$ animals were generated by inserting an IRES-GFP-pGK-Hygro ${ }^{r}$ cassette into the BamH1 site present in the 3' UTR of the Dbx1 gene. Recombination was achieved in two steps using the I-Scel-induced gene replacement system ${ }^{49}$. All mice were bred in a C57BL6 background. Day of plug was considered E0.5.

\section{In situ hybridization and Immunohistochemistry}


Transverse $20 \mu \mathrm{m}$ sections were obtained using a cryostat (Leica). The methods for in situ hybridization (ISH) coupled with immunohistochemistry and for double-fluorescence ISH on cryosections have been described ${ }^{3}$. Evx1 and Vglut2 (Slc17a6) riboprobres were synthesized using a DIG RNA labeling kit (Roche) as specified by the manufacturer. For fluorescent immunohistochemistry, sections were incubated overnight at $4{ }^{\circ} \mathrm{C}$ with one or several of the following primary antibodies: anti-NK1R (Sigma S8305; 1:5000), rabbit anti-somatostatin (Peninsula Labs T-4103; 1:500), rabbit anti-GFP (Invitrogen A11122; 1:1000), chicken anti- $\beta$-Gal (abcam 9361; $1: 1000$ ), chicken anti-GFP (Aves Labs 1020; 1:2000), mouse anti-Islet1,2 (DSHB 39.4D5c; 1:250) and guinea-pig anti-Evx1 (gift from T.M. Jessell; 1:4000). The following secondary antibodies were used: donkey anti-rabbit Cy5 (Jackson IR 744-176-152; 1:500), goat anti-rabbit Alexa 594 (Invitrogen A11012; 1:500), goat anti-chicken Alexa 488 or 647 (Invitrogen A11039 and A21449 respectively; 1:500), goat anti-mouse FITC, Cy3 or Cy5 (Abcam 6945, 6563 and 6785-1; 1:500) and donkey anti-guinea-pig Сy3 (Jackson IR 706-166-148; 1:500). Biocytin was revealed using extravidin conjugated to either FITC or $\mathrm{Cy} 3$ at 1:400 final concentration during the secondary antibody incubation. Slides were scanned on a SP2 confocal microscope (Leica Microsystems). A contrast enhancement and a noise reduction filter were applied under Adobe Photoshop to images.

\section{Definition of the preBötC area and cellular counts}

The embryonic preBötC area is defined using previous physiological and anatomical criteria ${ }^{32,50}$ that were confirmed in the present study. Spontaneous rhythmic activity characterizing the preBötC is present in transverse slice preparations in a domain ventral and medial to the n.a. that is fully circumscribed by a $300 \mu \mathrm{m} \times 300 \mu \mathrm{m}$ square positioned such that the ventral aspect of the n.a. is set in the dorsolateral quadrant (Figs $3, \mathbf{4}$ ). Rhythmic activity was systematically detected at the rostral surface of $450 \mu \mathrm{m}$-thick transverse medullary slices respectively set $250 \mu \mathrm{m}$ and $300 \mu \mathrm{m}$ posterior to the caudal limit of the facial nucleus in E15.5 and E18.5 embryos. These slices encompass a 300-350 $\mathrm{\mu m}$ longitudinal column of the ventrolateral medulla presenting highest density of interneurons showing somatic and cellular processes expressing high level of $\mathrm{NK}_{1} \mathrm{R}^{+}$at these stages and at E18.5 highest density of $\mathrm{Sst}^{+}$neurons. Thus neuronal counts in the preBötC area were performed within the volume defined by the $300 \mu \mathrm{m} \times 300 \mu \mathrm{m}$ square area, having an anterior posterior extent of $300 \mu \mathrm{m}$ starting $250 \mu \mathrm{m}$ and $300 \mu \mathrm{m}$ caudal to the posterior limit of the facial motor nucleus respectively for E15.5 and E18.5 embryos.

For each marker, neurons were counted manually on raw images, with the help of the cell counter plug-in in ImageJ, in 3 to 9 cryosections from the preBötC area on one side, in 2 to 4 animals. Cellular counts per section from all animals were summed to calculate the proportions presented in Table 1 or were averaged per animal and a grand mean \pm s.e.m was calculated across animals to produce cells per section histograms summarizing anatomical stainings in Figures 3, 4, S1 
and S3. For cellular counting after retrograde tracing experiments using biocytin, physiological $450 \mu \mathrm{m}$ thick slices were frozen and re-sectioned in $20 \mu \mathrm{m}$ thick sections and retrogradely-filled neurons were counted from all sections. Then these numbers were averaged across preparations and are expressed in the text and Fig. $5 j, k, l$ as mean \pm s.e.m. per preBötC.

\section{In vitro preparations}

Pregnant mice were sacrificed by cervical dislocation on E15.5. Embryos were excised from the uterus and kept at $16-18{ }^{\circ} \mathrm{C}$ in artificial cerebrospinal fluid (aCSF) bubbled with carbogen (95\% O2, $5 \%$ CO2) until the electrophysiological and/or optical recordings sessions. aCSF composition in mM was: $120 \mathrm{NaCl}, 8$ $\mathrm{KCl}, 1.26 \mathrm{CaCl} 2,1.5 \mathrm{MgCl} 2,21 \mathrm{NaHCO}, 0.58 \mathrm{Na} 2 \mathrm{HPO} 4,30$ glucose, $\mathrm{pH}=7.4$. A high external $[\mathrm{K}+]$ was purposefully used to ensure maintenance of the functional mode of the preBötC previously described at the time of its emergence ${ }^{32}$. Whole hindbrain preparations and preBötC slices were prepared as previously described ${ }^{32}$. In brief, hindbrains attached to the first 6 segments spinal segments were isolated from the embryo and either transferred in a recording chamber, ventral side up, for recordings, or embedded in an agar block, mounted onto a vibratome (Leica) and serially sectioned in the transverse plane from rostral to caudal until the posterior limit of the facial nucleus was reached. Using this as landmark we further removed a 200 to $250 \mu \mathrm{m}$ thick slices and then performed the $450 \mu \mathrm{m}$ thick physiological slice that exposes the preBötC at its rostral surface. Both en bloc preparations and slices were kept at $30{ }^{\circ} \mathrm{C}$ in a recording or injection chamber and continuously superfused with oxygenated aCSF. Prior to any recordings or injection, preparations were allowed to recover for $30 \mathrm{~min}$.

\section{Electrophysiological recordings}

C4 nerve root activity in whole hindbrain preparations and population activity of the preBötC on transverse slices were recorded using glass micropipettes suction electrodes (150 $\mu \mathrm{m}$ tip diameter) filled with aCSF and connected through silver wires to a high-gain AC amplifier (Grass, 7P511). The signal was filtered (bandwidth: $3 \mathrm{~Hz}-3 \mathrm{k} \mathrm{Hz}$ ), integrated using an electronic filter (Neurolog System, time constant $100 \mathrm{~ms}$ ), recorded on a computer via a digitizing interface (Digidata 1322A; Molecular Devices, Sunnyvale, CA, USA) and analyzed with the pClamp9 software (Molecular Devices).

Whole-cell patch-clamp neuronal recordings of GFP-expressing cells were performed on transverse slices using a E-600-FN upright microscope (Nikon, Tokyo, Japan) equipped with a standard epifluorescent illumination system and a Coolsnap HQ CCD camera (Photometrics, Tucson, AZ). After identification of a $\mathrm{GFP}^{+}$cell in the area of the preBötC, whole-cell recording was achieved under visual control using differential interference contrast (DIC). Patch electrodes (resistance of 4 to $6 \mathrm{M} \Omega$ ) were pulled from borosilicate glass tubes (Clark GC 150TF, Pangbourn, UK) and filled with a solution containing (in $\mathrm{mM}$ ): $123 \mathrm{~K}$ gluconic acid, $21 \mathrm{KCl}, 0.5$ EGTA, $3 \mathrm{MgCl}$, 10 HEPES, pH 7.2, supplemented with 
$1 \mathrm{mg} / \mathrm{ml}$ biocytine (Molecular Probes, Eugene, OR). Electrophysiological signals were recorded using an Axopatch200B amplifier (Molecular Devices), a digitizing interface (Digidata 1322A, Molecular Devices) and the software program pClamp9 (Molecular Devices). Substance $P(S P)$ and the $\mu$-opiates agonist DAMGO were obtained from Sigma, dissolved in aCSF and bath-applied for 10 to 15 minutes at the final concentration of $0.1 \mu \mathrm{M}$. Values are given as means \pm SEM and statistical significance was tested using unpaired difference Student's $t$ test. Differences were regarded as significant if $p<0.05$.

\section{Calcium imaging}

Whole hindbrain and slices were incubated for $40 \mathrm{~min}$ in oxygenated aCSF containing the cell-permeable calcium indicator dye Calcium-Green 1AM. After a 30 min recovery period in the recording chamber to wash out the dye excess, whole hindbrain preparations were positioned in the recording chamber with the ventral side up. Fluorescence images were captured with a cooled CCD camera (Coolsnap HQ, Photometrics, Tucson, AZ) using an exposure time of $100 \mathrm{~ms}$ in overlapping mode (simultaneous exposure and readout) mounted on an E-600FN upright microscope (Nikon) equipped with a standard epi-fluorescent illumination system. A fluorescein filter set was used to excite the dye and capture the emitted light, during periods of 60 to $180 \mathrm{~s}$. Images were analyzed using Metamorph software (Universal Imaging Corporation, West Chester, PA). To perform calcium imaging of GFP expressing cells, we first acquired images of GFP cells together with corresponding DIC images. After dye loading, we carefully re-positioned over the same cellular field by aligning cellular profiles with the DIC image stored prior to incubation. The GFP-labeled cells false colored red image (Fig. 2h, top) and the calcium-loaded cells false colored green image (Fig. $2 \mathrm{~h}$, bottom) were overlayed to determine double labeled somas and to position regions of interest for measurements of fluorescence changes (encircled cells in Fig. 2h). In all cases, the average intensity in a region of interest was calculated for each frame and changes in fluorescence were normalized to their initial value by expression as the ratio of changes in fluorescence to initial fluorescence $(\Delta F / F)$.

\section{Plethysmographic recordings}

E18.5 fetuses delivered from pregnant dams (Dbx1 ${ }^{\text {Lacz/+ }}$ in-crossings) or P0 neonates were placed inside an enclosed $30 \mathrm{ml}$ plethysmographic chamber for 90 seconds. Chamber temperature was taken before and after the recording. Ventilation was measured by recording pressure fluctuations, relative to a reference chamber of corresponding size, that are proportional to tidal volume. Data were sampled at $1 \mathrm{KHz}$. Respiratory frequency was calculated on a breathto-breath basis using whole body plethysmography (Buxco Electronics Inc., NY, USA) and analysed using Elphy (UNIC, CNRS).

\section{Retrograde labeling of commissural neurons in the preBötC}


Biocytin was dissolved at $2 \mathrm{mg} / \mathrm{ml}$ in distilled water containing $2 \%$ DMSO and a few $\mu \mathrm{g}$ of Fast-Green FCF (Sigma) to obtain a solution visible under standard transmitted light illumination. Using broken-tip patch clamp micropipette (tip diameter 10-20 $\mu \mathrm{m}$ ), the solution was pressure injected during 45 minutes onto the preBötC area on medullary transverse slices continuously superfused with oxygenated aCSF of the same composition as above. A larger micro-pipette (tip diameter 100-200 $\mu \mathrm{m}$ ) connected to a pump was placed above the injection site to continuously absorb any dye overspill thus ensuring its focal application. After the injection, micropipettes were removed and the slice was maintained in the chamber for at least 1 hour to allow intracellular diffusion of the dye, then it was fixed in $4 \%$ PFA at $4{ }^{\circ} \mathrm{C}$ for 2 hours, then rinsed in PBS and immuno-processed. For cellular counting, the $450 \mu \mathrm{m}$ slices were cryoprotected in $30 \%$ sucrose, frozen, re-sectioned into $20 \mu \mathrm{m}$ sections and processed for immunohistochemistry as described above.

\section{REFERENCES}

1. Feldman, J.L. \& Del Negro, C.A. Looking for inspiration: new perspectives on respiratory rhythm. Nat Rev Neurosci 7, 232-242 (2006).

2. Thoby-Brisson, M., et al. Genetic identification of an embryonic parafacial oscillator coupling to the preBotzinger complex. Nature neuroscience 12, 10281035 (2009).

3. Dubreuil, V., et al. Defective respiratory rhythmogenesis and loss of central chemosensitivity in Phox $2 \mathrm{~b}$ mutants targeting retrotrapezoid nucleus neurons. J Neurosci 29, 14836-14846 (2009).

4. Rose, M.F., Ahmad, K.A., Thaller, C. \& Zoghbi, H.Y. Excitatory neurons of the proprioceptive, interoceptive, and arousal hindbrain networks share a developmental requirement for Math1. Proceedings of the National Academy of Sciences of the United States of America 106, 22462-22467 (2009).

5. Smith, J.C., Ellenberger, H.H., Ballanyi, K., Richter, D.W. \& Feldman, J.L. Pre-Botzinger complex: a brainstem region that may generate respiratory rhythm in mammals. Science 254, 726-729 (1991).

6. Gray, P.A., Rekling, J.C., Bocchiaro, C.M. \& Feldman, J.L. Modulation of respiratory frequency by peptidergic input to rhythmogenic neurons in the preBotzinger complex. Science 286, 1566-1568 (1999).

7. Stornetta, R.L., et al. A group of glutamatergic interneurons expressing high levels of both neurokinin-1 receptors and somatostatin identifies the region of the pre-Botzinger complex. J Comp Neurol 455, 499-512 (2003).

8. Tan, W., Pagliardini, S., Yang, P., Janczewski, W.A. \& Feldman, J.L. Projections of preBotzinger Complex neurons in adult rats. The Journal of comparative neurology 518, 1862-1878 (2010).

9. Gray, P.A., Janczewski, W.A., Mellen, N., McCrimmon, D.R. \& Feldman, J.L. Normal breathing requires preBotzinger complex neurokinin-1 receptorexpressing neurons. Nature neuroscience 4, 927-930 (2001).

10. Tan, W., et al. Silencing preBotzinger complex somatostatin-expressing neurons induces persistent apnea in awake rat. Nature neuroscience 11,538540 (2008). 
11. Blanchi, B., et al. MafB deficiency causes defective respiratory rhythmogenesis and fatal central apnea at birth. Nature neuroscience 6, 10911100 (2003).

12. Wallen-Mackenzie, A., et al. Vesicular glutamate transporter 2 is required for central respiratory rhythm generation but not for locomotor central pattern generation. J Neurosci 26, 12294-12307 (2006).

13. Koizumi, H., et al. Functional imaging, spatial reconstruction, and biophysical analysis of a respiratory motor circuit isolated in vitro. J Neurosci 28, 2353-2365 (2008).

14. Koshiya, N. \& Smith, J.C. Neuronal pacemaker for breathing visualized in vitro. Nature 400, 360-363 (1999).

15. Jen, J.C., et al. Mutations in a human ROBO gene disrupt hindbrain axon pathway crossing and morphogenesis. Science (New York, N.Y 304, 1509-1513 (2004).

16. Marillat, V., et al. The slit receptor Rig-1/Robo3 controls midline crossing by hindbrain precerebellar neurons and axons. Neuron 43, 69-79 (2004).

17. Renier, N., et al. Genetic dissection of the function of hindbrain axonal commissures. PLoS biology 8, e1000325 (2010).

18. Sabatier, C., et al. The divergent Robo family protein rig-1/Robo3 is a negative regulator of slit responsiveness required for midline crossing by commissural axons. Cell 117, 157-169 (2004).

19. Zhang, Y., et al. V3 spinal neurons establish a robust and balanced locomotor rhythm during walking. Neuron 60, 84-96 (2008).

20. Jessell, T.M. Neuronal specification in the spinal cord: inductive signals and transcriptional codes. Nat Rev Genet 1, 20-29 (2000).

21. Lumsden, A. \& Krumlauf, R. Patterning the vertebrate neuraxis. Science

274, 1109-1115 (1996).

22. Briscoe, J. \& Ericson, J. Specification of neuronal fates in the ventral neural tube. Curr Opin Neurobiol 11, 43-49 (2001).

23. Garcia-Campmany, L., Stam, F.J. \& Goulding, M. From circuits to behaviour: motor networks in vertebrates. Current opinion in neurobiology 20, 116-125.

24. Lanuza, G.M., Gosgnach, S., Pierani, A., Jessell, T.M. \& Goulding, M. Genetic identification of spinal interneurons that coordinate left-right locomotor activity necessary for walking movements. Neuron 42, 375-386 (2004).

25. Briscoe, J., et al. Homeobox gene Nkx2.2 and specification of neuronal identity by graded Sonic hedgehog signalling. Nature 398, 622-627 (1999).

26. Pattyn, A., Hirsch, M.-R., Goridis, C. \& Brunet, J.-F. Control of hindbrain motor neuron differentiation by the homeobox gene Phox2b. Development 127, 1349-1358 (2000).

27. Pattyn, A., et al. Coordinated temporal and spatial control of motor neuron and serotonergic neuron generation from a common pool of CNS progenitors. Genes Dev 17, 729-737 (2003).

28. Pattyn, A., Vallstedt, A., Dias, J.M., Sander, M. \& Ericson, J.

Complementary roles for Nkx6 and $\mathrm{Nkx} 2$ class proteins in the establishment of motoneuron identity in the hindbrain. Development 130, 4149-4159 (2003).

29. Pierani, A., et al. Control of interneuron fate in the developing spinal cord by the progenitor homeodomain protein Dbx1. Neuron 29, 367-384 (2001).

30. Pierani, A., Brenner-Morton, S., Chiang, C. \& Jessell, T.M. A sonic hedgehog-independent, retinoid-activated pathway of neurogenesis in the ventral spinal cord. Cell 97, 903-915 (1999). 
31. Ericson, J., et al. Pax6 controls progenitor cell identity and neuronal fate in response to graded Shh signaling. Cell 90, 169-180 (1997).

32. Thoby-Brisson, M., Trinh, J.B., Champagnat, J. \& Fortin, G. Emergence of the pre-Botzinger respiratory rhythm generator in the mouse embryo. J Neurosci 25, 4307-4318 (2005).

33. Funk, G.D., Smith, J.C. \& Feldman, J.L. Generation and transmission of respiratory oscillations in medullary slices: role of excitatory amino acids. Journal of neurophysiology 70, 1497-1515 (1993).

34. Rekling, J.C. \& Feldman, J.L. PreBotzinger complex and pacemaker neurons: hypothesized site and kernel for respiratory rhythm generation. Annu Rev Physiol 60, 385-405 (1998).

35. McKay, L.C., Janczewski, W.A. \& Feldman, J.L. Sleep-disordered breathing after targeted ablation of preBotzinger complex neurons. Nature neuroscience $\mathbf{8}$, 1142-1144 (2005).

36. Liu, Y.Y., et al. Relationship between two types of vesicular glutamate transporters and neurokinin-1 receptor-immunoreactive neurons in the preBotzinger complex of rats: light and electron microscopic studies. The European journal of neuroscience 17, 41-48 (2003).

37. Del Negro, C.A., Johnson, S.M., Butera, R.J. \& Smith, J.C. Models of respiratory rhythm generation in the pre-Botzinger complex. III. Experimental tests of model predictions. J ournal of neurophysiology 86, 59-74 (2001). 38. Johnson, S.M., Koshiya, N. \& Smith, J.C. Isolation of the kernel for respiratory rhythm generation in a novel preparation: The pre-Botzinger complex "island". J ournal of neurophysiology 85, 1772-1776 (2001).

39. Bianchi, A.L., Denavit-Saubie, M. \& Champagnat, J. Central control of breathing in mammals: neuronal circuitry, membrane properties, and neurotransmitters. Physiol Rev 75, 1-45 (1995).

40. Moran-Rivard, L., et al. Evx1 is a postmitotic determinant of v0 interneuron identity in the spinal cord. Neuron 29, 385-399 (2001).

41. Geisen, M.J., et al. Hox paralog group 2 genes control the migration of mouse pontine neurons through slit-robo signaling. PLoS biology 6, e142 (2008). 42. Wilson, S.I., Shafer, B., Lee, K.J. \& Dodd, J. A molecular program for contralateral trajectory: Rig-1 control by LIM homeodomain transcription factors. Neuron 59, 413-424 (2008).

43. Srour M, R.J., Pham JM, Dubé MP, Girard S, Morin S, Dion PA, Asselin G, , Rochefort D, H.P., Diab S, Sharafaddinzadeh N, Chouinard S, Théoret H, Charron \& F, R.G. Mutations in DCC cause congenital mirror movements. Science 328(5978), 592 (2010).

44. Kiehn, O. Locomotor circuits in the mammalian spinal cord. Annu Rev Neurosci 29, 279-306 (2006).

45. Morgado-Valle, C., Baca, S.M. \& Feldman, J.L. Glycinergic pacemaker neurons in preBotzinger complex of neonatal mouse. J Neurosci 30, 3634-3639 (2010).

46. Cheng, L., et al. TIX3 and TIX1 are post-mitotic selector genes determining glutamatergic over GABAergic cell fates. Nature neuroscience 7, 510-517 (2004). 47. Bielle, F., et al. Multiple origins of Cajal-Retzius cells at the borders of the developing pallium. Nature neuroscience 8, 1002-1012 (2005).

48. Keller, C., Hansen, M.S., Coffin, C.M. \& Capecchi, M.R. Pax3:Fkhr interferes with embryonic Pax3 and Pax7 function: implications for alveolar rhabdomyosarcoma cell of origin. Genes \& development 18, 2608-2613 (2004). 
49. Cohen-Tannoudji, M., et al. I-SceI-induced gene replacement at a natural locus in embryonic stem cells. Molecular and cellular biology 18, 1444-1448 (1998).

50. Bouvier, J., et al. Brain-derived neurotrophic factor enhances fetal respiratory rhythm frequency in the mouse preBotzinger complex in vitro. The European journal of neuroscience 28, 510-520 (2008). 
Dbx1

E10.5
B-Gal

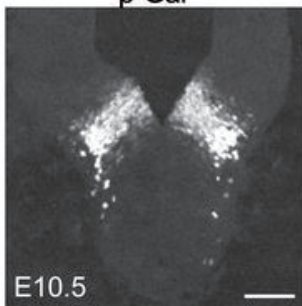

B-Gal Is 1 .2

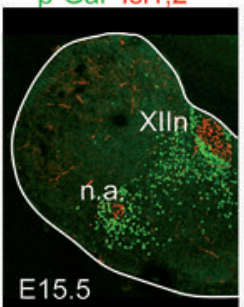

$\Delta F / F$

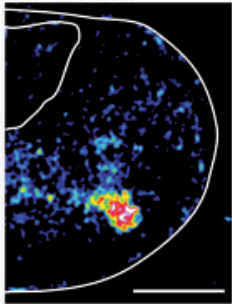

$D b \times 1$ iresGFP

$\mathrm{Db} \times 1$

E10.5

\section{GFP}

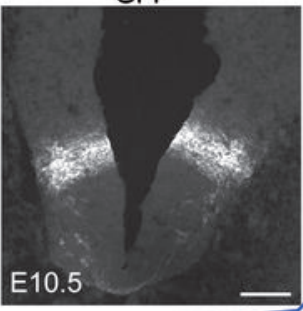

\section{GFP}

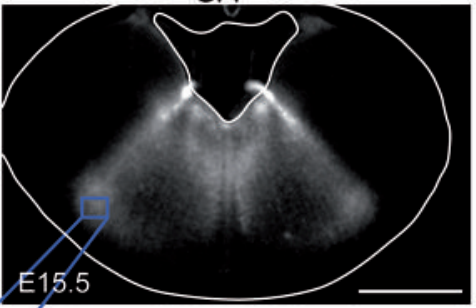

E10.5

Merge

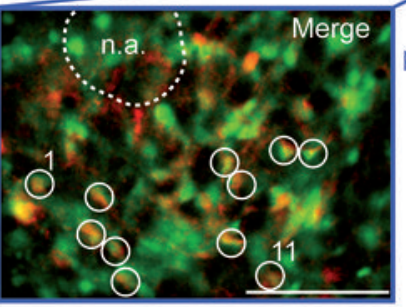

preBötC Cell $1+2)$ Wand in

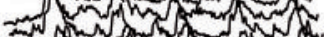
Now the,

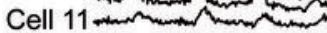

-


- Transverse slice
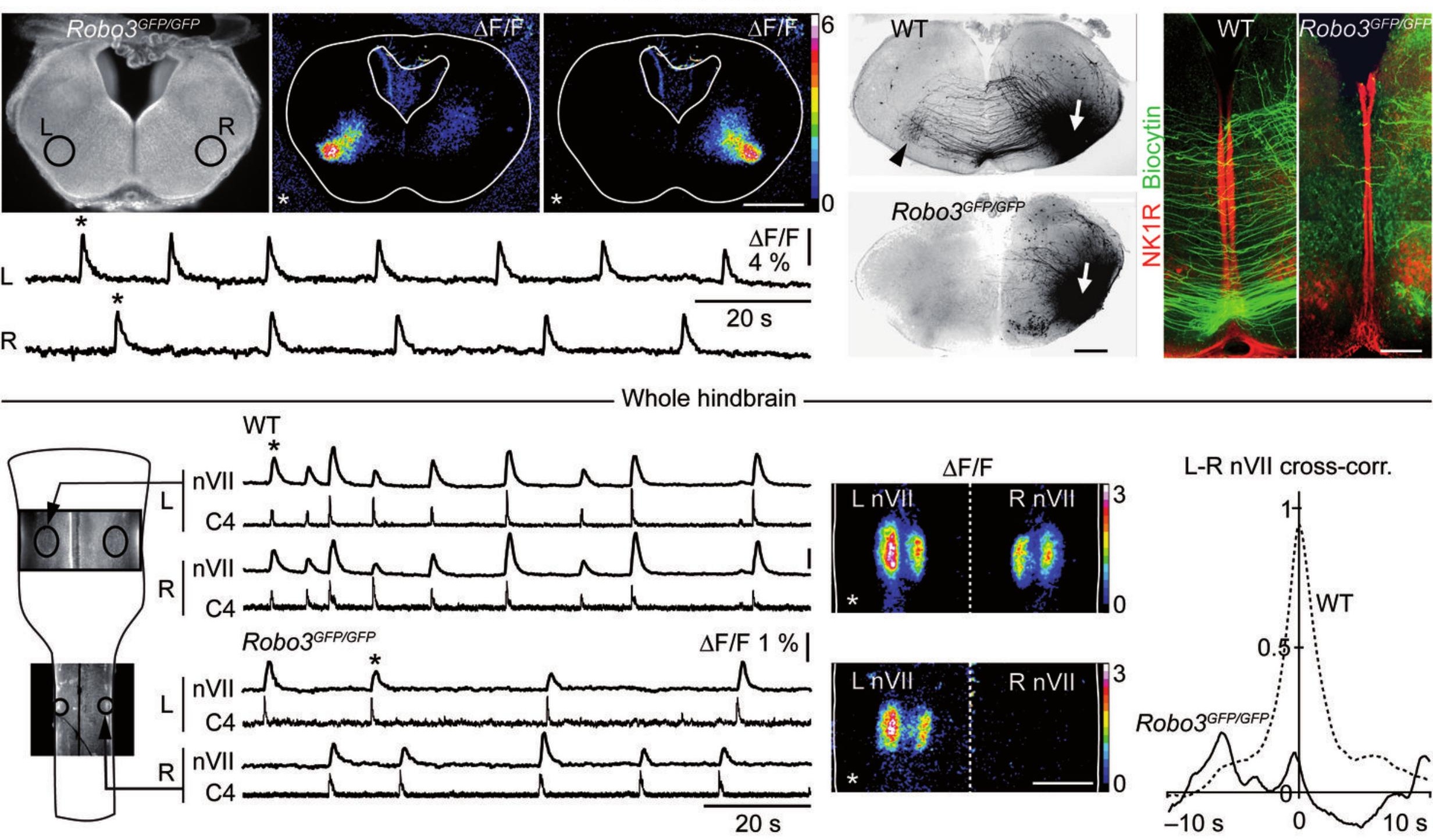

L-R nVII cross-corr.
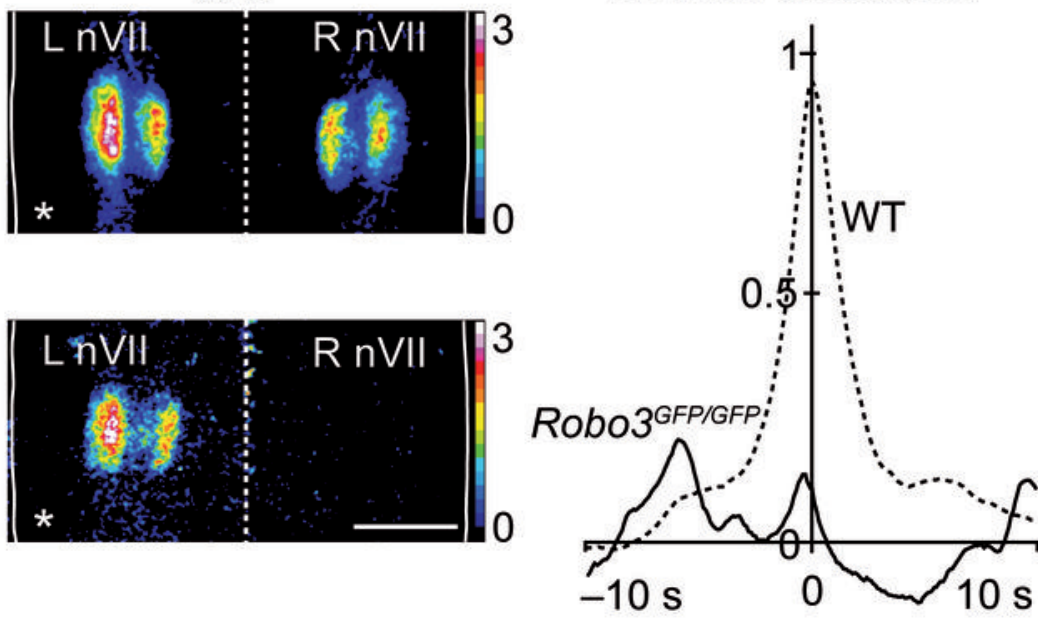
L Int.
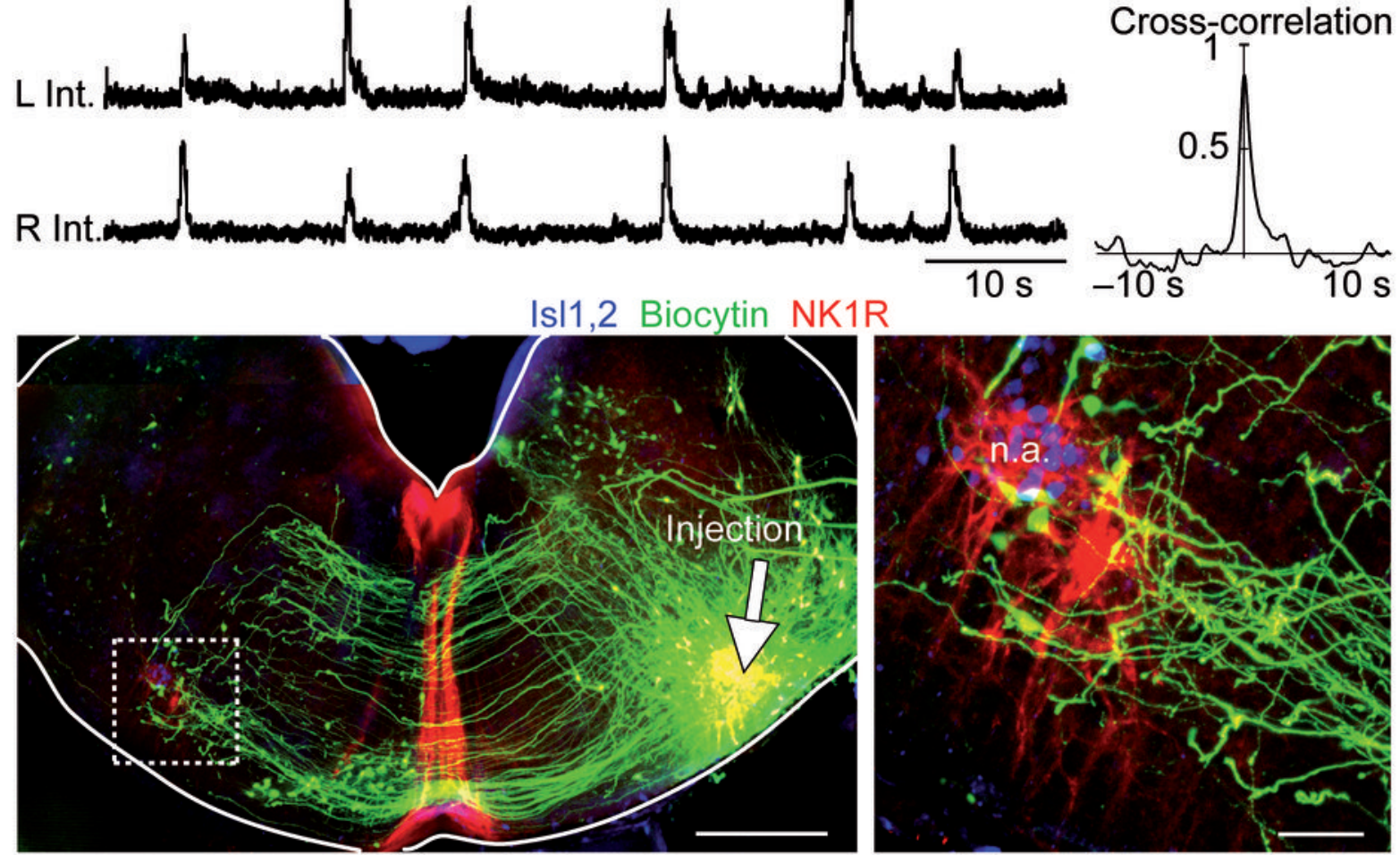

Dbx1::Cre;Robo3 $3^{10 \times 10 x}$

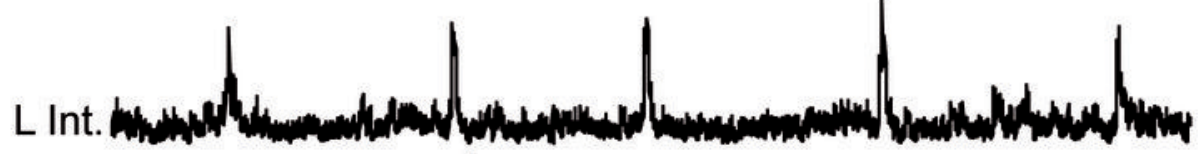

Cross-correlation

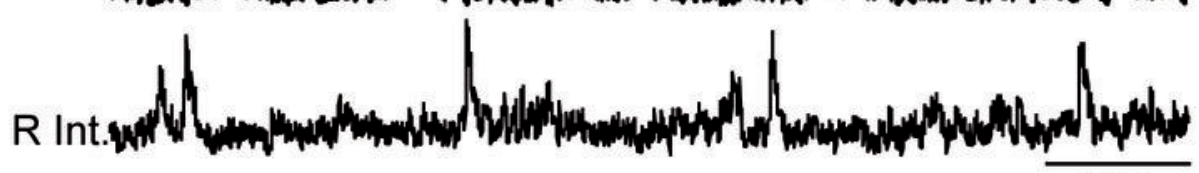
Is|1,2 Biocytin NK1R
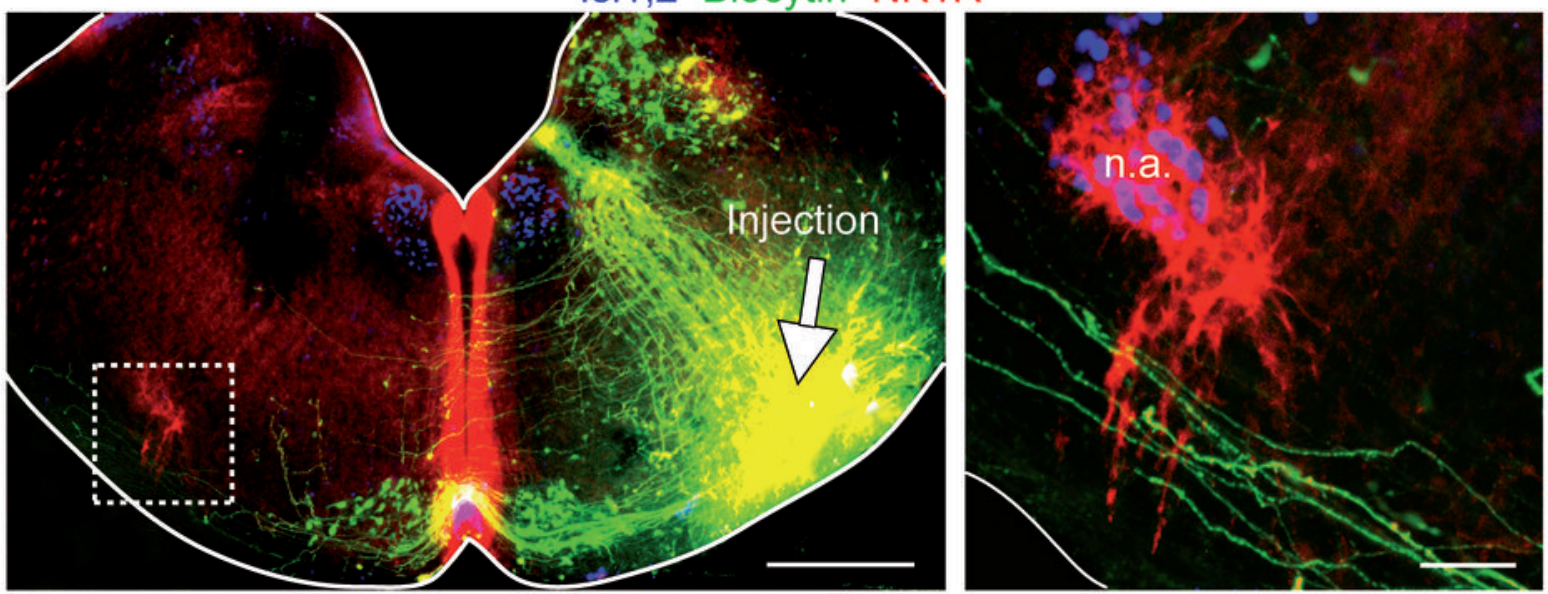